\title{
Análisis del estado de la capa activa en el emplazamiento de la base antártica española Gabriel de Castilla, Isla Decepción, Antártida
}

\author{
M.A. de Pablo(1), A. Molina(1), C. Recio(2), M. Ramos(3), G. Goyanes ${ }^{(4)}$ y M.A. Ropero(5) \\ (1) Unidad de Geología. Dpto. de Geología, Geografía y Medio Ambiente. Universidad de Alcalá. \\ 28871 Alcalá de Henares, Madrid. España. \\ miguelangel.depablo@uah.es \\ (2) Dpto. de Matemática Aplicada a las TIC. Universidad Politécnica de Madrid, 28040 Madrid. España.
} (3) Unidad de Física. Dpto. de Física y Matemáticas. Universidad de Alcalá. 28871 Alcalá de Henares, Madrid. España. (4) Instituto Antártico Argentino - Dirección Nacional del Antártico / CONICET. San Martín, Provincia de Buenos Aires. Argentina.

(5) Laboratorio de Ingenieros del Ejército "General Marvá" - INTA. Ministerio de Defensa. 28008 Madrid. España.

\section{RESUMEN}

La degradación de los suelos congelados (permafrost) y el aumento de espesor de la capa activa pueden deberse tanto a factores naturales, como antrópicos (edificaciones, carreteras, cimentaciones, etc.) que modifican las condiciones naturales y, por tanto, sus características termo-mecánicas, tal y como ha sido profusamente estudiado en el hemisferio Norte. En el caso de la Antártida, algunas bases se encuentran en zonas de permafrost, como es el caso de la base española Gabriel de Castilla en la isla Decepción. En la zona donde se localiza esta base se ha observado un importante aumento de la erosión, con la excavación de torrenteras y el retroceso del escarpe costero. Con la hipótesis de la degradación del permafrost como causa principal, se decidió evaluar los posibles efectos de la base (su módulo principal) en el estado de la capa activa y el permafrost sobre el que se asienta. Para ello, entre 2012 y 2015 se han tomado distintas medidas de temperatura del suelo, espesor de la capa activa y de temperatura en el interior del edificio y bajo la base, cuyos resultados se presentan y analizan en este trabajo. Se concluye que la edificación ejerce un doble efecto, reduciendo el enfriamiento del terreno durante el invierno, en el que permanece cerrada, $y$ facilitando su calentamiento durante los meses de actividad, durante el verano antártico. Estos resultados hacen que sea necesario un estudio de las causas y se tomen las medidas y acciones necesarias para reducir los efectos de la degradación de la capa activa bajo la base, así como una investigación sistemática del estado de la capa activa y el permafrost en la zona afectada por la presencia de la base antártica.

Palabras clave: Antártida, erosión, movimiento en masa, periglaciarismo, permafrost

\section{Study of the active layer at the Spanish Antarctic station "Gabriel de Castilla", Deception Island, Antarctica}

\begin{abstract}
The degradation of permanent frozen ground (permafrost) and the increase in the thickness of the active layer may be caused both by natural processes (such as global climate change) and by anthropic activity, which changes the natural environmental conditions that allow its existence, as has been widely reported to occur in the northern polar and subpolar regions. In the case of Antarctica, some scientific research stations are located in areas with permafrost, such as the Spanish Antarctic station "Gabriel de Castilla" on Deception Island. In the place where the station is located, an important increase in erosion has been observed in recent years, including the excavation of new gullies and the erosion of the coastal cliffs. In order to develop an initial analysis of the possible effects of the station on the permafrost degradation, ground temperature has been monitored since 2012 and the thickness and of the active layer and the temperature, both inside and beneath the station, have also been sporadically measured. Here we show the results and discuss how the station reduces the freezing of the ground during the winter when the station is closed and
\end{abstract}


de Pablo, M.A., et al., 2017. Análisis del estado de la capa activa en el emplazamiento... Boletín Geológico y Minero, 128 (1): $69-92$

facilitates the warming of the ground during the living periods of the station in the Antarctic summer. Those initial results and conclusions make necessary to continue the study of the permafrost and the active layer in the station site by systematic monitoring of the ground temperature and the thickness of the active layer.

Keywords: Antarctica, erosion, mass wasting, periglacial, permafrost

\section{ABRIDGED ENGLISH VERSION}

\section{Introduction and methods}

Since 2010, an increase in erosion has been observed at the site where the Spanish Antarctic station "Gabriel de Castilla" (BGdC) is located on Deception Island, Antarctica (Fig. 1). This erosion is evident from the increase in the depth of the gullies, as well as the excavation of new ones and the erosion of the nearby coastal cliffs $15-20$ m downslope in front of the main module of the station. The increasing erosion may have natural causes on periglacial regions where permafrost exists. Global warming could thaw the frozen ground, thus increasing the thickness of the active layer, allowing more water to flow and, therefore, produce erosion on the surface. This has been reported to occur in mountain regions and also more widely in the northern polar territories. However, human activity might also cause the permafrost to thaw and the thickness of the active layer to increase, as has been widely reported to occur related to constructions in the northern polar and subpolar territories. In order to know if the BGdC could be also causing the melting of the permafrost and the consequent increase in the surface erosion, we initiated a monitoring programme of the thermal state of the permafrost and the evolution of the thickness of the active layer in February 2012, with the first results shown here. We also began to study the active layer and permafrost in the location of the station at the same time, mainly related with the main module (living and dormitory accommodation areas; Fig. 2).

The area where is the station located (Fig. 3) is characterized by pyroclastic materials from the last volcanic eruptions in the volcano, corresponding to the post-caldera Pendulum Cove Formation, and the consequent volcanoclastic materials formed from its erosion, mainly by fluvial, glacial and coastal processes. From the geomorphological point of view, the station lies on a low slope glacis deposit located at the base of a hill (62.8 $\mathrm{m}$ a.s.l.) and $15-20 \mathrm{~m}$ from the coastal scarps. Those characteristics allow us to apply similar research techniques to those we have been using in Antarctica since the late 1980's to study permafrost and active layers following the international monitoring protocols from Ground Thermal State-Permafrost (GTS-P) and Circumpolar Active Layer Monitoring (CALM) of the International Permafrost Association (IPA). We measured the active layer thickness by mechanical probing in February 2012 in the settlement of the base, and especially at the perimeter of the main module of the base (Fig. 4). We drilled a shallow borehole (70 cm deep) close to the main module which contains an array of miniature temperature devices (DS1922L iButton model from Maxim Co.) which have been taking data every 4 hours all the year around, since early 2012. Throughout 10 days in late 2014 and early 2015, indoor/outdoor temperature measurements were taken at the main building of the base, at different sites (communications centre, living room, kitchen, bathroom, and dorms). We also used the iButton devices, taking data every hour. Outdoor sensors were joined to $20 \times 20$ $\mathrm{cm}$ metallic plates and buried at less than $1 \mathrm{~cm}$ to avoid direct heating. Finally, we carried out a GPS-D survey of the area to allow us to explain the data (Fig. 4).

\section{Results and discussion}

The analysis of the thickness of the active layer, ground thermal evolution and indoor/outdoor temperature monitoring at the BGdC between February 2012 and January 2015 shows a slight increase in the freezing period duration and a consequent reduction in the duration of the thawing period (Fig. 5), in a similar way to that we observed in other sites of the island and also in other islands of the South Shetland archipelago.

The active layer thickness derived from these ground temperature data for the 2012-2014 period is about $50 \mathrm{~cm}$ (Fig. 6; Table 1) although the mean annual temperature of the ground in the upper $60 \mathrm{~cm}$ is warmer than $-0.5^{\circ} \mathrm{C}$ (Fig. 6). This means that the permafrost is very unstable and sensitive to any change in the surface environmental conditions, including temperature changes and inter-annual snow cover variations.

The thickness of the active layer measured by mechanical probing in 2012 is about $15 \mathrm{~cm}$ at the base, although it quickly increases towards the nearest coast. However, the thickness of the active layer measured in the perimeter of the main module of the base shows values of $45 \mathrm{~cm}$, which could increase up to about $90 \mathrm{~cm}$ in selected sites (Fig.7). 
de Pablo, M.A., et al., 2017. Análisis del estado de la capa activa en el emplazamiento... Boletín Geológico y Minero, 128 (1): $69-92$

Inside and outside temperatures monitored for a 10-day period in late 2014 and early 2015 showed that while the terrain follows the daily fluctuations of the air temperatures, the ground below the station shows this pattern less clearly and is strongly smoothed, but it does show patterns which correspond to indoor temperatures at the main module of the station (Fig. 8). Although there is not a direct correlation between indoor and outdoor temperatures (Fig. 9), these observations lead us towards a connection between them. The heating index calculated for the different monitoring locations (Table 2), and their correlations regarding the more outdoor temperatures is evidence of this connection between indoor and outdoor temperatures which is different, depending on the site where the temperature is measured in this module (Fig. 10).

From all these first results, we are able to conclude that the ground below the main module of the $B G d C$ does not have the same behaviour due to the shield effect of the building. In spite of the edifice not being in direct contact with the surface, the inner heat seems to be transmitted in some way to the ground, may be partially due to the inadequate insulation of the floor of the module. Moreover, the shield effect of the edifice does not allow the normal freezing of the ground during the winter. Therefore, despite the fact that the snow-free period is reducing, as revealed by the thermal data from the borehole, the ground is not frozen enough to compensate the heat from the floor of the station during the summer, when the station is open.

This combination of these effects could, therefore, be responsible for the increase in the thickness of the active layer we observe in our data. The degradation of the permafrost has other collateral damage, because the increase of the snow cover layer reported for the last few winters implies an increase in the available water during the thawing period, when the active layer is not frozen. Then, the runoff water is able to excavate and erode the pyroclastic materials of the slope on which the base is located, allowing the formation of new gullies or increasing the dimensions of the older ones. The consequences of this process have been observed during the summer in early 2015, when an important gully exposed some important infrastructures of the base, and formed new deep gullies in the slope and apron deposits on the coast (Fig.11).

\section{Conclusions}

We conclude that the main module of the Spanish Antarctic station "Gabriel de Castilla" has a negative influence on the natural behaviour of the permafrost and the active layer, causing the thawing of the permafrost and the increasing of the active layer due to the shield effect of the environmental conditions, with the help of the heating from the edifice itself when it is operative during the summer seasons. This scenario explains how an increasing thickness of the active layer leaves a thicker pyroclastic layer, which is easily eroded by runoff. This runoff also seems to be increasing due to the elongation of the snow cover period and its thickness, as has been reported since 2010. Based on these conclusions, we propose continuing this research in more depth by starting further systematic monitoring of the permafrost and the active layer at this site on Deception Island.

\section{Introducción}

En las regiones del planeta donde las condiciones climáticas son lo suficientemente frías (regiones polares, subpolares y de alta montaña), el terreno puede congelarse durante el invierno, descongelándose durante el periodo estival, definiendo lo que se denominan suelos de congelación estacional. En ocasiones, cuando los suelos no llegan a descongelarse completamente se denominan suelos permanentemente congelados (permafrost). Así, la definición clásica de permafrost es la de los terrenos que permanecen con temperaturas por debajo de los $0^{\circ} \mathrm{C}$ durante, al menos, dos años consecutivos (Muller, 1943; Linell y Tedrow, 1981; Harris et al., 1988; Van Everdingen, 1998). Sin embargo, aunque no todo el suelo se descongele, la parte superficial suele hacerlo para volver a congelarse durante el invierno siguiente. A esa parte del terreno más superficial situada por encima del permafrost, que está sometida a ciclos de congelación y descongelación periódicos, se la denomina capa activa, y tiene una gran importancia desde el punto de vista ecológico, hídrico y geomorfológico. Por lo tanto, la capa activa es la única zona del terreno hasta una cierta profundidad con temperaturas positivas durante una parte del año. La existencia de agua líquida en la capa activa y el efecto cíclico de los periodos de congelación/descongelación permiten que el agua intersticial pueda fluir, favoreciendo el movimiento de materiales, y permitiendo así el desarrollo de vida en ambientes en principio poco favorables. Este proceso también genera formas del relieve muy características, como los suelos ordenados, 
de Pablo, M.A., et al., 2017. Análisis del estado de la capa activa en el emplazamiento... Boletín Geológico y Minero, 128 (1): $69-92$

lóbulos de solifluxión, entre otros (ej. Oht, 2003; French, 2007).

Sin embargo, el espesor de la capa activa y su gradiente de temperatura pueden verse incrementados si el balance de energía en la superficie del suelo es positivo, como está ocurriendo en la actualidad por efecto del calentamiento global, y del que la Antártida es un claro ejemplo (ej., Steig et al., 2009; Bromwich et al., 2013). El incremento del espesor de la capa activa y su temperatura conlleva que parte del permafrost que está situado inmediatamente por debajo de ella se vaya fundiendo, es decir, se vaya degradando. En algunos lugares del planeta, esta degradación del permafrost está permitiendo que la materia orgáni$\mathrm{ca}$, antes congelada, pueda descomponerse; hecho que da lugar a la emisión de gases de efecto invernadero que, a su vez, incrementan aún más el calentamiento global (Anisimov et al., 1997; Osterkamp, 2003). Desde el punto de vista aplicado a la ingeniería civil, este proceso de degradación del permafrost tiene también grandes efectos directos, como la pérdida de resistencia y cohesión del terreno, algo que en zonas habitadas supone un riesgo geológico importante por colapso de infraestructuras, edificios, canalizaciones, etc. Esto ha hecho que se desarrollen numerosos estudios dirigidos a evitar la degradación de los suelos congelados, ya sea por efectos naturales o derivados de la actividad antrópica (ej., Péwé, 1983; Williams, 1986; Andersland y Ladanyi, 1994; U.S. Arctic Research Commission Permafrost Task Force, 2003; Zhao-Ping et al., 2010; Schoeneich et al., 2011). La aplicación de las técnicas constructivas desarrolladas para reducir y/o evitar la descongelación del suelo en los pilares que asientan las estructuras, que se proponen en esos trabajos ha tenido también su aplicación en regiones menos habitadas como la Antártida, donde el incremento del espesor de la capa activa y la consiguiente degradación del permafrost ha tenido lugar estos últimos años como efecto del calentamiento (ej., Guglielmin y Vieira, 2014; Guglielmin et al., 2014; Oliva y Ruiz-Fernández, 2015). Aunque en la Antártida no existen zonas habitadas como tales, las bases de investigación científica han ido adaptando sus diseños arquitectónicos a la necesidad de reducir su impacto sobre los suelos congelados, incorporando las infraestructuras revestimientos con perfiles fluidodinámicos elevados que minimizan la resistencia frente a los vientos, favoreciendo así un mínimo impacto sobre la configuración original del terreno (ej. Ropero, 2013). Y es que actividades que producen el calentamiento del suelo o la eliminación de la cubierta nival al final del invierno, favorecen la descongelación del terreno, lo que acaba causando la degradación del permafrost con la consiguiente reducción de la resistencia mecánica del suelo y el hundimiento de los pilares de las estructuras. En el caso de la Antártida, estos posibles procesos asociados a las instalaciones son una afección sobre el medio ambiente en un territorio protegido por el Tratado Antártico (ATS, 1959) y su anexo medioambiental, el Protocolo de Madrid (ATS, 1991).

En el caso de la base Antártica Española "Gabriel de Castilla" (a partir de aquí, BGdC), desde el año 2009 se venía observando el deterioro de algunas partes de la edificación, así como el incremento de los procesos erosivos en el terreno alrededor de la base. Esto podía hacer sospechar la degradación del permafrost, en parte relacionada con la existencia de la propia edificación del módulo principal de la misma. Entre otros efectos de menor entidad, el incremento del número y profundidad de las cárcavas existentes en la ladera sobre la que se ubica la base, especialmente las situadas frente al módulo principal de la misma, fue el motivo por el que durante la campaña antártica 2011-2012, se decidió estudiar el estado de la capa activa y el permafrost en el entorno de la base, especialmente en el perímetro del módulo principal. En febrero de 2012 se decidió iniciar el control del estado térmico y evolución del permafrost y la capa activa en el entorno del módulo principal de la BGdC, de forma complementaria a los estudios que se venían realizando sobre el permafrost y la capa activa en la isla Decepción (ej., Ramos et al., 1989, 2007, 2012; Vieira et al., 2010). El interés del estudio de la capa activa en este lugar ha sido recientemente respaldado por las recomendaciones plasmadas en el informe final resultante de la inspección del Tratado Antártico realizada el pasado 31 de diciembre de 2014 a esta base antártica (ATS, 2015), en el que se recomienda a España el estudio del permafrost en dicho lugar dado el incremento de la erosión observada en la zona donde se ubica. Si bien este problema de incremento de la erosión podría tener diversas causas, como la propia acción de la banquisa y la erosión costera sobre el terreno, se estableció como hipótesis de partida que el permafrost posiblemente jugaba un papel importante en estos eventos de erosión del terreno y de afección a la edificación principal de la BGdC, dejando otras causas y sus soluciones ingenieriles como objetivo principal de otros trabajos de investigación (Recio, 2015). El objetivo del presente trabajo es mostrar los primeros resultados de las medidas de espesor de la capa activa y del comportamiento térmico del suelo en el entorno del módulo principal de la mencionada base para caracterizar el permafrost y la capa activa, y establecer el posible papel que su degradación podría estar jugando en el incremento de la erosión en el lugar $y$, por ende, sobre la propia BGdC. 


\section{Zona de estudio}

\section{Situación geográfica}

La zona de estudio se limita al entorno de la BGdC (62 $\left.58^{\circ} 38^{\prime \prime} \mathrm{S}, 60^{\circ} 40^{\prime} 21^{\prime \prime} \mathrm{O}\right)$, con una extensión de unos $10000 \mathrm{~m}^{2}$ que abarca la veintena de módulos que componen la base (Figura 1). Esta zona se localiza en la
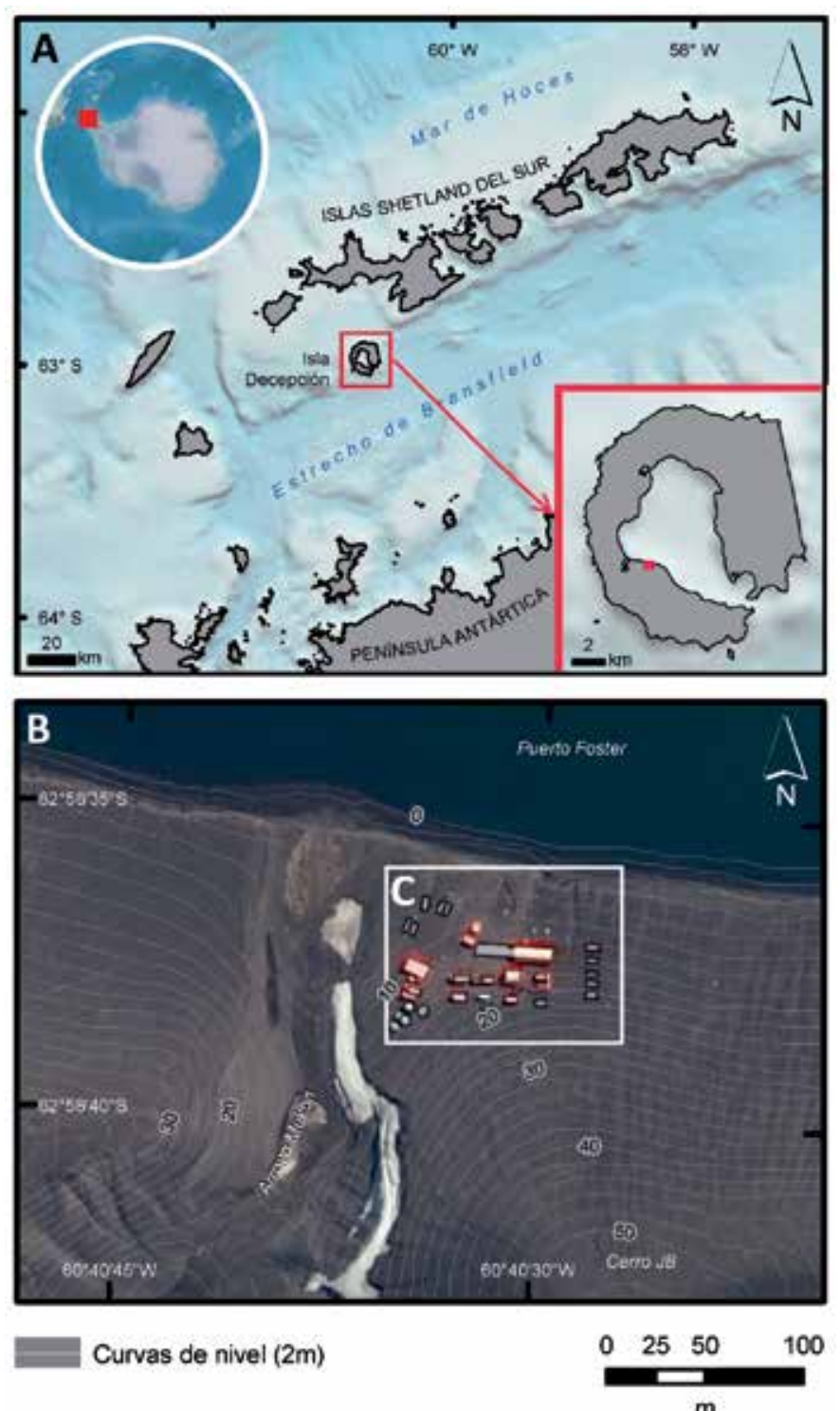

Figura 1. (A) Localización de la base Antártica Española Gabriel de Castilla en la costa interior de la isla Decepción, en el archipiélago de las islas Shetland del Sur, en la Antártida. (B) Vista de satélite con superposición de curvas de nivel de la zona de emplazamiento de la base y (C) delimitación de la zona de estudio.

Figure 1. (A) Location of the "Gabriel de Castilla" Spanish Antarctic Station at the inner coast of Deception Island, South Shetland Archipelago, in Antarctica. (B) Satellite image overlapped with topographic contour lines (equidistance $2 \mathrm{~m}$ ) of the station location site, and $(C)$ the studied area. isla Decepción, una isla de planta aproximadamente circular de unos $18 \mathrm{~km}$ de diámetro máximo que forma parte del archipiélago de las Islas Shetland de Sur, localizado a unos $110 \mathrm{~km}$ de la Península Antártica (separado de ésta por el Mar de Bransfield) y a unos 1000 $\mathrm{km}$ del extremo sur del continente suramericano.

En detalle (Figura 1), la zona de estudio se ubica al pie del monte en el que se ubica el vértice geodésico BEGC, a 62,80 m s.n.m., en una ladera de aproximadamente un $12 \%$ de pendiente orientada al norte y constituida por materiales piroclásticos y separada de la costa por un escarpe de 2-3 $\mathrm{m}$ de altura que da paso a una estrecha franja de playa. Esta costa se corresponde con el mar interior de la isla, denominado Puerto Foster, formado por una caldera volcánica inundada, rodeada por los restos del edificio volcánico colapsado, a modo de muralla anular de hasta 540 m s.n.m. de altitud en el Monte Pond y los 452 m s.n.m. en el Monte Kirkwood. El límite occidental de la zona de estudio está definido por los escarpes de 4-5 metros dejados por la erosión fluvial en el valle existente.

La isla se encuentra dentro del territorio del Tratado Antártico (ATS, 1959), firmado por España en el año 1982. La isla Decepción incluye varias Zonas Antárticas Especialmente Protegidas (ZAEP) debido a que en ellas se concentran áreas de gran relevancia natural, científica o histórica (ATS, 2012). Además, la isla ha sido designada Zona Antártica Especialmente Administrada (ZAEA).

\section{La base Gabriel de Castilla}

La actual BGdC (Figuras 1c y 2a) es el resultado de las consecutivas ampliaciones de las primeras instalaciones ubicadas en este lugar de la isla Decepción, que se iniciaron como un campamento de tiendas de campaña en el verano austral de 1989, durante el que se instalaron una serie de módulos agrupados que constituyeron el denominado refugio Gabriel de Castilla, ya a principios de 1990. El incremento de las necesidades de capacidad de alojamiento para personal científico así como de laboratorios hizo necesario realizar una ampliación de la base en la campaña 2001-2002, levantándose un nuevo módulo de vida (dormitorios, salón-comedor, aseos, cocina y centro de comunicaciones), y convirtiendo el antiguo refugio en el actual módulo científico de la base. Las diversas ampliaciones realizadas en la base desde aquel momento, dieron lugar a un complejo de módulos auxiliares, alrededor de una veintena en la actualidad, para el almacenamiento de víveres y materiales, combustible, generadores de electricidad y una incineradora, destacando además la ampliación 

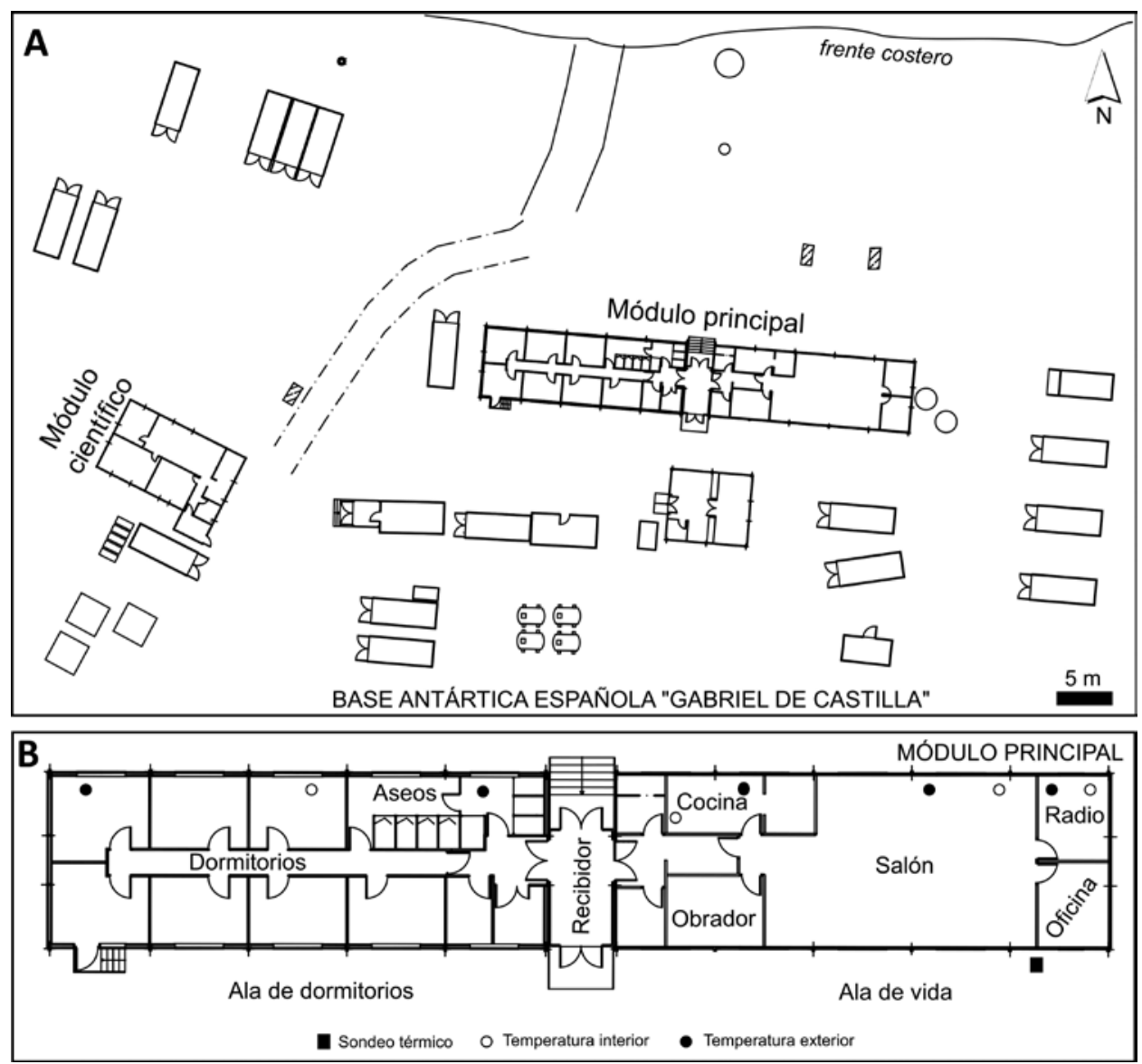

Figura 2. (A) Plano de la zona de estudio en torno al módulo principal de la base Gabriel de Castilla, y (B) detalle de la distribución interna de dicho módulo. Se indica la situación del sondeo térmico para control de temperaturas de la capa activa y el permafrost.

Figure 2. (A) Floor Plan of the study area showing the different modules of the "Gabriel de Castilla" Station, and (B) detail of the inner distribution in its main building, showing the location of the permafrost and active layer temperature monitoring sites.

del módulo principal (El módulo de principal es el módulo de vida y el módulo de alojamiento) durante las campañas antárticas 2008-2009 y 2009-2010. De esta forma, en la actualidad la BGdC está constituida por el módulo principal y el módulo científico, así como esa veintena de módulos auxiliares (Figura 2a).

El módulo principal de la base (Figura 2b), dividido en el ala de vida y el ala de dormitorios, es en realidad dos módulos de construcción independiente conectados por un distribuidor central. Estos módulos son barracones del modelo TYCE 6,5/92 mejorados, de $18,5 \mathrm{~m} \times 6,5 \mathrm{~m}$ cada uno (Ropero, 2010a, 2010b). Los pilares que transmiten las cargas de la estructura al terreno se apoyan en el ala de vida sobre tacos de madera, mientras que en el ala de dormitorios lo hacen sobre pequeñas zapatas de hormigón in situ. La altura de estos pilares es variable, ya que la fachada sur del módulo se encuentra a ras de la superficie, o ligeramente por debajo de ella (debido a la acumulación de materiales arrastrados a lo largo de los años por la arroyada difusa durante el deshielo), mientras que en la fachada norte, situada ladera abajo y mirando al frente costero, tienen una altura de entre $50 \mathrm{~cm}$ y algo más de $1 \mathrm{~m}$. La estructura de ambos brazos del módulo no es exactamente igual, pero en general están elevados del suelo mediante pilares de acero galvanizado, que soportan una estructura metálica en retícula sobre la que se apoyan los pilares y el sistema de cerchas de la cubierta (Ropero, 2010a, 2010b). El solado está compuesto por tablero aglomerado hidrófugo, con una barrera de vapor de chapa grecada de aluminio, un film de polietileno y una loseta de PVC de $6 \mathrm{~mm}$ de espesor. El cerramiento vertical consiste en paneles formados por una plancha de poliestireno expandido de $40 \mathrm{~mm}$ entre un tablero de aglomerado de $10 \mathrm{~mm}$ de espesor plastificado formando el paramento interior y una chapa de aluminio gofrado a la cara exterior. En su parte interior tiene lana de fibra de vidrio IBR-80, con barrera de vapor de una lámina de aluminio, terminada con un panel 
de Pablo, M.A., et al., 2017. Análisis del estado de la capa activa en el emplazamiento... Boletín Geológico y Minero, 128 (1): $69-92$

aglomerado de $22 \mathrm{~mm}$ acabado en melanina. La carpintería exterior es de aluminio lacado con rotura de puente térmico y doble acristalamiento estanco. La cubierta se compone de un panel sándwich formado en sus caras exteriores por chapa de acero galvanizado lacado al horno, de espesor $0,6 \mathrm{~mm}$ y con $4 \mathrm{~cm}$ de aislamiento de poliuretano.

\section{Clima}

La isla Decepción se caracteriza por tener un clima frío, clasificado (según la clasificación de Koppen, 1918) como polar marítimo templado -Cfc- (King y Turner, 1997) o tundra -ET- (Peel et al., 2007). Los datos meteorológicos recogidos en la estación de la Agencia Estatal de Meteorología en dicha base desde el año 2005 indican una temperatura media anual desde ese año que ronda los $-2^{\circ} \mathrm{C}$, con máximas registradas de $12,7^{\circ} \mathrm{C}$ y mínimas de $-22,5^{\circ} \mathrm{C}$. Las humedades relativas son de en torno a $80-90 \%$. Las precipitaciones medias son de unos $990 \mathrm{~mm}$ anuales, principalmente en forma de nieve, aunque se han llegado a registrar hasta $23 \mathrm{~mm}$ de lluvia en algunos veranos australes (Bañón y Vasallo, 2015).

Aunque el interior de la isla se encuentra protegido de los vientos predominantes del Sureste por los relieves montañosos que forman la caldera del volcán, la apertura de los Fuelles de Neptuno en el anillo de la isla permite la entrada al interior de la misma de vientos de componente Sur, cuya velocidad media es de unos $25 \mathrm{~km} / \mathrm{h}$, pero con rachas fuertes siempre inferiores a los $160 \mathrm{~km} / \mathrm{h}$ (Bañón y Vasallo, 2015).

Las precipitaciones de nieve en la isla Decepción, al igual que en la próxima isla Livingston, se producen generalmente desde marzo hasta noviembre. Tras eso es cuando suele comenzar la fusión de la nieve, aunque en ocasiones se ha detectado que permanecen numerosos parches de nieve hasta bien entrado el verano, durante el mes de febrero en algunos emplazamientos. Las mayores precipitaciones sólidas ocurren en otoño, entre los meses de septiembre y noviembre, con espesores totales medidos en lugares cercanos a la base de alrededor de 1-1,5 m (ej. Jiménez, 2013), aunque en el emplazamiento de la base se han llegado a observar mantos de nieve de hasta 4 metros de altura al final del invierno, debido a la precipitación y al viento. En esto último influye la localización de la BGdC y la distribución de los edificios ejerciendo efecto pantalla frente a los vientos costeros. La fusión de esta nieve conlleva un aporte extra de agua de escorrentía, que, como se verá a continuación da lugar a algunos de los rasgos geomorfológicos observados, al combinarse con la erosionabilidad de los materiales volcánicos en la zona de estudio.

\section{Geología y geomorfología}

El terreno sobre el que se asienta la BGdC es de naturaleza volcánica, ya que la isla Decepción es un volcán activo. Las últimas erupciones en la isla tuvieron lugar entre 1967 y 1970, y aún hoy en día persiste una importante actividad geotermal, fumarólica y sísmica (Ibañez et al., 2003; Almendros et al., 2004). Su origen está relacionado con un sistema de fracturas en el fondo oceánico, a más de $850 \mathrm{~m}$ bajo la superficie, de dirección SW-NE en el llamado Arco de Scotia (Hawkes, 1962; Baker et al., 1975; Grad et al., 1992; Rey y Somoza, 1992; Rey et al., 1995; González-Casado et al., 2000; Somoza et al., 2004; Maestro et al., 2007). Todos los materiales presentes en la isla tienen un origen volcánico, hidrotermal o volcanoclástico por la modificación de los dos primeros (Smellie, 2001, 2002).

En la zona concreta de estudio, donde se ubica la BGdC, destacan dos tipos de materiales (Figura 3). Uno de ellos es la Formación Caleta Péndulo, que está constituida por rocas piroclásticas formadas durante erupciones hidrovolcánicas explosivas post-caldera, es decir, formadas posteriormente a la importante depresión interna que caracteriza la isla, hoy ocupada por el mar en el denominado Puerto Foster. Este tipo de materiales es uno de los más extendidos por la superficie de la isla, como se muestra en el mapa geológico de la misma (Smellie y López-Martínez, 2000). El espesor de estos materiales en la zona de estudio es indeterminado.

La otra unidad geológica existente en la zona de estudio es un conjunto de materiales de origen volcanoclástico formados por la erosión y removilización de la unidad anterior por diferentes causas. Esto incluye procesos de ladera, solifluxión, fluviales y costeros. Son, por tanto, materiales de granulometría variable, predominando el tamaño lapilli, aunque en zonas situadas a mayor altitud esta llega a tamaño bloque y con carácter más anguloso. Desde el punto de vista geotécnico, los materiales superficiales de esta unidad (sobre la que se asienta la BGdC) son arenas limosas, con contenido en gravas variable, permeabilidad muy baja y capacidad drenante baja (LIE-Ropero, 2015).

Desde el punto de vista geomorfológico (Figura 3) existen mapa geomorfológico 1:25000 de la isla Decepción (López-Martínez et al., 2000), la descripción geomorfológica acompañante (López-Martínez y Serrano, 2002) y una cartografía geomorfológica 


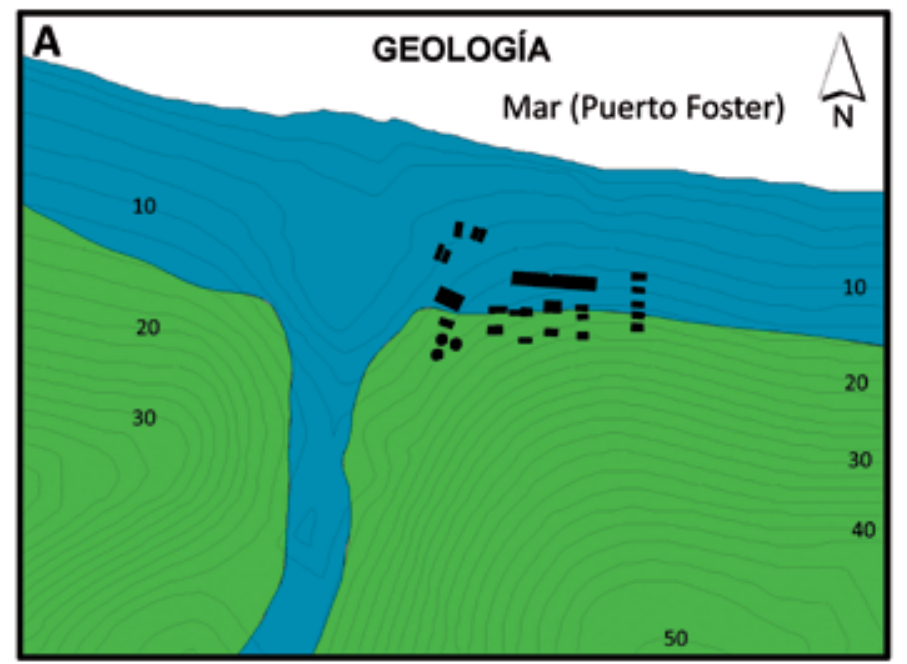

Cuaternario indeferenciado

Fm. Caleta Péndulo

Módulos BAE

Curvas de nivel (2m)

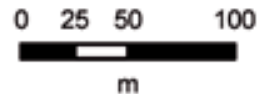

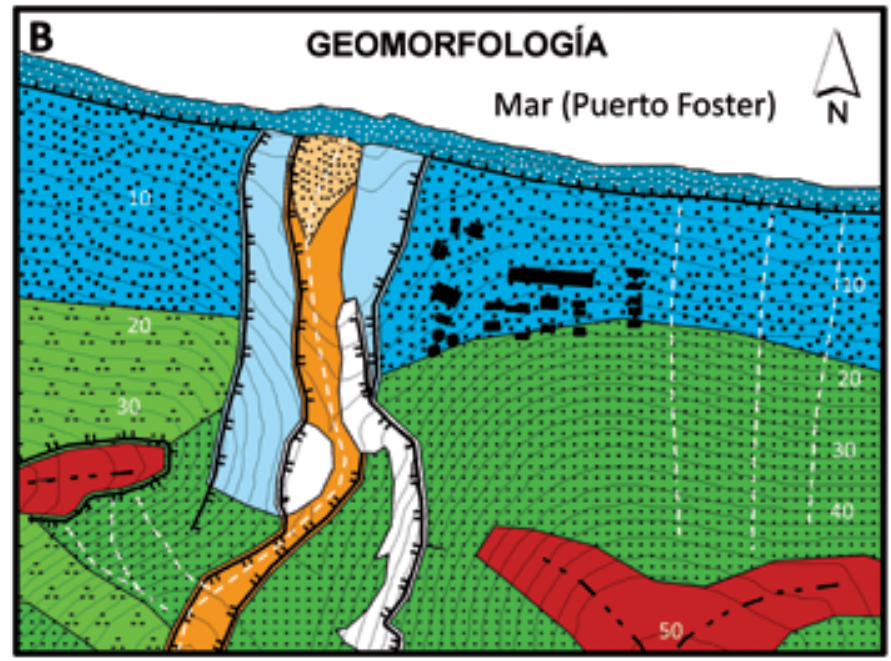
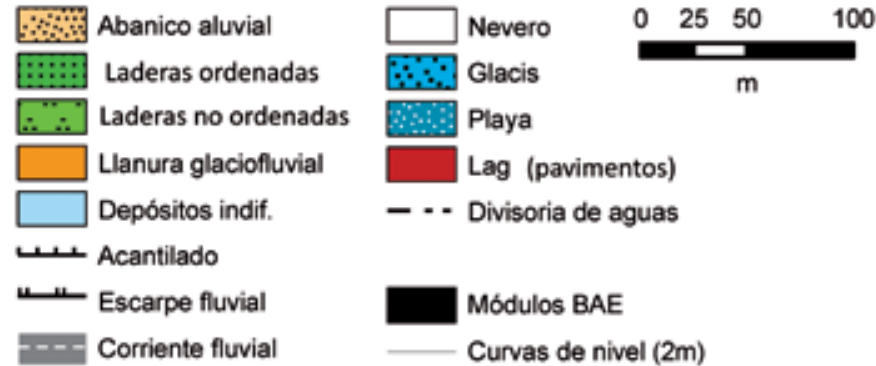

Figura 3. Esquemas geológico (A) y geomorfológico (B) de la zona estudiada en la isla Decepción (modificado de Goyanes, 2015). Figure 3. Geological (A) and geomorphological (B) sketch maps of the study site on Deception Island (modified from Goyanes, 2015).

más reciente a escala 1:15000 (Goyanes, 2015). En estos trabajos destaca que la isla esté cubierta en más de un $50 \%$ de su superficie por glaciares, por lo que la actividad glacial es un importante agente modelador de su relieve. Sin embargo, la zona de estudio se encuentra libre de glaciares, destacando otros procesos como principales agentes modeladores. Esta zona está dominada por glacis de acumulación en la parte inferior de la ladera, formados por el aporte de los sedimentos desde ésta con procesos de escorrentía en el caso de individualizarse cursos efímeros y/o por aquellas laderas con procesos de gelifluxión en su ausencia. En las cotas más elevadas, los suelos pavimentados (lag), originados por la erosión de los vientos, que arrastran los materiales piroclásticos más finos (deflacción). En la sección inferior, próximo a las aguas de Puerto Foster se reconoce un acantilado de 2-3 m de altura, en cuya base se desarrolla una playa con material de tamaño lapilli. Inmediatamente al oeste de la base se encuentra una superficie plana, limitada en ambos extremos por escarpes fluviales que disminuyen su altura hacia la costa clasificándose como una planicie glaciofluvial. Sobre ella se desarrollan en la época estival cursos fluviales provenientes de la fusión de los glaciares ubicados sobre el monte Kirkwood. Asimismo es posible identificar pequeños abanicos aluviales en sectores donde dos de estos cursos se unen. Sobre los escarpes fluviales de mayor altura se produce la acumulación de potentes espesores de nieve, algunos de los cuales han permanecido durante varios veranos en forma de neveros.

Debido a las condiciones climáticas, los terrenos libres de hielo de la isla Decepción, incluyendo los costeros como el de la ubicación de la BGdC, se congelan durante el invierno. Los primeros centímetros se descongelan durante la época de verano austral, debido principalmente al balance de la radiación solar sobre la superficie del suelo (alcanzando el espesor de la capa activa su máximo anual al final del verano), mientras que el terreno en profundidad permanece congelado de manera permanente (permafrost). Los estudios de control del permafrost que se realizan de forma continua en la isla han mostrado que aunque existen zonas de permafrost discontinuo, éste puede alcanzar más de $5 \mathrm{~m}$ de espesor, como 
en los emplazamientos situados en la zona del Cráter Lake, en las proximidades de la BGdC (Hauk et al., 2007; Goyanes et al., 2014a), con un máximo espesor anual de la capa activa de aproximadamente $30 \mathrm{~cm}$. De forma general, y como ocurre en otros puntos del planeta a altas latitudes, es esperable que el espesor de la capa activa aumente a medida que nos acercamos a la costa debido al efecto atemperador que ejerce el mar. Por tanto, los materiales volcanoclásticos que se encuentran en la zona de estudio se ven fuertemente cementados por el hielo durante el invierno, mientras que durante el verano las zonas más superficiales se descongelan. Esta fusión se produce fundamentalmente por efecto del balance de radiación solar combinada con el efecto de la ablación del agua de fusión de la nieve acumulada, que llega en las proximidades de la base a $1.5 \mathrm{~m}$ de espesor máximo anual, pero que dado el efecto pantalla de los módulos de la base, alcanza más de $3 \mathrm{~m}$ a sotavento en algunos puntos irregularmente distribuidos.

\section{Métodos y obtención de datos}

La toma de datos para desarrollar este estudio ha sido realizada a lo largo de cuatro campañas antárticas entre febrero de 2012 y enero de 2015. Alguno de los métodos presentados a continuación no han podido aplicarse de forma repetida a lo largo de este periodo de estudio debido a las limitaciones logísticas de trabajar en este emplazamiento remoto, pero también por la variable presencia de nieve durante los periodos de trabajo (que se extendieron en etapas irregulares entre los meses de diciembre y enero).

Este estudio comprende distintas aproximaciones metodológicas tomadas del clásico estudio de los suelos congelados, tal y como se realiza dentro de los protocolos internacionales del estudio térmico de los suelos en ambientes glaciares y periglaciares (Thermal State of Permafrost - TSP) y de la monitorización de la capa activa (Circumpolar Active Layer Monitoring - CALM) (ej., Brown et al., 2000; Matsuoka y Humlum, 2003; Nelson et al., 2004; Matsuoka, 2006), dentro de la red de estudio de la temperatura del suelo permanentemente congelado (Ground Temperature Network Permafrost - GNT-P) englobado en la Asociación International del Permafrost (International Permafrost Association - IPA).

Con el objetivo de determinar la profundidad del permafrost y el espesor de la capa activa, así como establecer sus características térmicas, en febrero de 2012 se realizó una perforación de $70 \mathrm{~cm}$ de profundidad junto a la pared sur del ala de vida del módulo principal de la BGdC (Figura 2; Figura 4). Este sondeo, de $40 \mathrm{~mm}$ de diámetro, está entubado y sellado en ambos extremos, conteniendo 7 sensores digitales miniaturizados de temperatura situados a 2,5, 10, 20,40 y $70 \mathrm{~cm}$ de profundidad. Los sensores empleados son de tipo iButton (Maxim), modelo DS1922L con $0,5^{\circ} \mathrm{C}$ de precisión (aunque en la práctica se ha establecido que dicha precisión puede llegar a ser de $0,2^{\circ} \mathrm{C}$; ej., Gubler et al., 2011), y $0.0625^{\circ} \mathrm{C}$ de resolución, que toman datos cada 3 horas, con memoria para 4096 medidas (Figura 4).

Con los datos obtenidos ha sido posible calcular la presencia de nieve en la superficie a partir del estudio de la amplitud térmica diaria del sensor situado

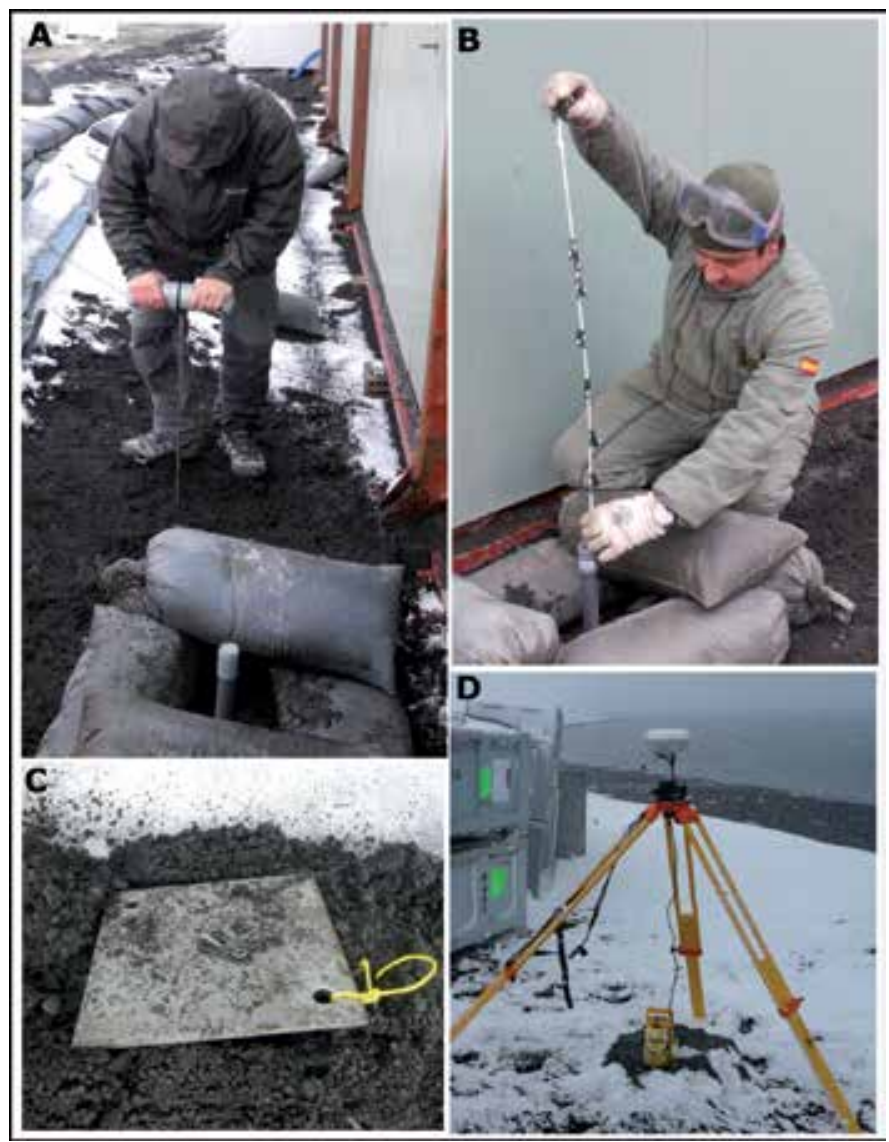

Figura 4. (A) Sondeos mecánicos manuales para la media del espesor de la capa activa; (B) Sondeo térmico que contiene una cadena de registradores miniaturizados de temperatura; (C) Placas isotermas de $20 \times 20 \mathrm{~cm}$ para la media de la temperatura bajo la base y en su interior; (D) Antena de GPS diferencial empleada para el levantamiento topográfico.

Figure 4. (A) Mechanical probing of the active layer; (B) shallow boreholes including an array of miniature temperature sensors, such as those used $(C)$ isothermal plates $(20 \times 20 \mathrm{~cm})$ to monitor inside and outside temperatures in the main module of the Station. (D) GPS-D antena used for the topographic survey. 
de Pablo, M.A., et al., 2017. Análisis del estado de la capa activa en el emplazamiento... Boletín Geológico y Minero, 128 (1): $69-92$

más cerca de la misma (ej., Zhang, 2005; Danby y Hik, 2007; Lewkowicz, 2008; Gadek y Leszkiewicz, 2010). Estos mismos datos son empleados para el cálculo de la temperatura máxima, media y mínima anual para cada profundidad, pudiendo realizarse perfiles de gradiente de temperatura que permiten determinar la profundidad del techo del permafrost (por definición, la profundidad a la cual la curva de la temperatura máxima anual sobrepasa la isoterma de los $0^{\circ} \mathrm{C}$ ), o su aproximación mediante la interpolación y extensión en profundidad de la curva de las temperaturas máximas anuales (ej. de Pablo et al., 2014).

Por otro lado, también en febrero de 2012, se realizó la medición de la profundidad del frente de descongelación como aproximación a la profundidad de la capa activa. Esta profundidad se midió mediante sondeos mecánicos manuales consistentes en la introducción de una barra de acero de $1 \mathrm{~cm}$ de diámetro y $1 \mathrm{~m}$ de longitud en el terreno hasta alcanzar el terreno congelado (Figura 4), siguiendo los mencionados protocolos internacionales (Brown et al., 2000; Matsuoka y Humlum, 2003; Nelson et al., 2004; Matsuoka, 2006), cuyo error es inferior a $1 \mathrm{~cm}$. Estas mediciones fueron realizadas en el entorno del módulo de vida de la BGdC a distancias crecientes desde el exterior de la misma, así como en los alrededores más próximos. Estos datos han sido establecidos mediante el uso de un dispositivo de posicionamiento global (GPS Garmin 76), posteriormente integrados en un Sistema de Información Geográfica (SIG) mediante el uso del programa gratuito de código abierto Quantum GIS en su versión 2.10.1 (www. qgis.org). Estos datos han sido interpolados mediante triangulación de los vecinos próximos con el fin de generar un mapa de la distribución de la profundidad del frente de descongelación. Debido a la presencia de nieve en las siguientes campañas antárticas, no fue posible la repetición de estas medidas para estudiar su evolución.

Complementariamente, en diciembre de 2014 se realizó un levantamiento topográfico RTK mediante un dispositivo GPS-diferencial Trimble modelo R6 (Figura 4D), especialmente enfocado a la realización de perfiles topográficos representativos de toda la ladera que caracteriza la zona de estudio. Estos datos fueron integrados en el mencionado SIG.

Finalmente, con el fin de determinar el posible efecto térmico en el terreno por el calentamiento de la BGdC, en diciembre de 2014 también se realizaron medidas de la temperatura del interior del módulo de vida, así como del suelo bajo la base en ubicaciones cercanas a las de los sensores en el interior del módulo (Figura 2). Se instalaron 4 sensores en el interior, expuestos al aire y siempre a menos de 30 cm de altura sobre el suelo del módulo; así como 6 sensores en el exterior, instalados cada uno de ellos sobre una placa metálica de $20 \times 20 \times 1 \mathrm{~cm}$, enterrada a $1 \mathrm{~cm}$ de profundidad para evitar el calentamiento directo por radiación solar (Figura 4). Uno de ellos fue instalado en un lugar próximo a la base, pero separado de ella (junto al mástil de banderas), con el fin de servir de referencia del comportamiento del suelo en condiciones no influenciadas por los edificios de la base. Los sensores empleados fueron igualmente de tipo iButton y modelo DS1922L, tomando datos cada hora. Las ubicaciones seleccionadas para los sensores (interiores y exteriores) fueron (Figura 2): centro de comunicaciones, salón, cocina, aseos y dormitorios. Los datos de estos sensores han permitido establecer diferencias y correlaciones térmicas entre el interior y el exterior de cada ubicación a lo largo de 10 días. Mediante ellos se ha realizado el cálculo de los índices de calentamiento, una variante de los índices de descongelación aquí empleada (ej., Andersland y Ladanyi, 1994), y que se ha calculado como la suma acumulada de los datos horarios de temperatura superior a $\operatorname{los} 0^{\circ} \mathrm{C}$.

\section{Resultados}

\section{Temperatura de la capa activa}

Los datos de temperatura obtenidos en el sondeo de la base (Tabla 1) muestran una evolución hacia la homogeneización a los $0^{\circ} \mathrm{C}$ a lo largo del periodo de estudio, tanto para las temperaturas de verano como las de invierno. También se aprecia una reducción del periodo en el que el terreno permanece libre de hielo en la superficie (Figura 5), pasando de 3 meses en 2013 a unos 15 días en 2014. En el año 2015 no se había registrado aún el inicio de dicho periodo, permaneciendo el sondeo cubierto por la nieve.

La máxima temperatura registrada en el periodo de estudio fue de unos $5^{\circ} \mathrm{C}$ en 2012 , reduciéndose hasta menos de $1^{\circ} \mathrm{C}$ en 2013. En cambio, durante el invierno, las temperaturas han ido ascendiendo en el terreno para acercarse a los $0^{\circ} \mathrm{C}$ durante mucho más tiempo a lo largo del año. Así, en el año 2012, las temperaturas en el terreno alcanzaron un mínimo de unos $-5^{\circ} \mathrm{C}$, mientras que en 2014 apenas llegaron a los $-2^{\circ} \mathrm{C}$, lo que se corresponde con una mayor y más prolongada presencia de nieve en la superficie.

Por otro lado, el cálculo de las temperaturas máximas, medias y mínimas registradas a cada profundidad en el sondeo, a lo largo del periodo de estudio de 3 años, ha permitido la realización del perfil térmico del terreno (Figura 6). En éste se observa una marcada 
de Pablo, M.A., et al., 2017. Análisis del estado de la capa activa en el emplazamiento... Boletín Geológico y Minero, 128 (1): $69-92$

\begin{tabular}{|l|c|c|c|c|c|c|c|c|c|c|c|c|}
\hline \multirow{2}{*}{$\begin{array}{l}\text { Prof. } \\
\text { (cm) }\end{array}$} & \multicolumn{3}{|c|}{$2012-2014$} & \multicolumn{4}{|c|}{2012} & \multicolumn{3}{c|}{2013} & \multicolumn{3}{c|}{2014} \\
\cline { 2 - 13 } & Máx. & Mín. & Media & Máx. & Mín. & Media & Máx. & Mín. & Media & Máx. & Mín. & Media \\
\hline 2,5 & 4,9 & $-4,5$ & $-0,30$ & 4,9 & $-4,5$ & 4,91 & 3,5 & $-2,4$ & 3,53 & 0,0 & 0,0 & $-0,04$ \\
\hline 5 & 4,0 & $-4,2$ & $-0,32$ & 4,0 & $-4,2$ & 4,02 & 2,6 & $-2,3$ & 2,64 & 0,9 & $-1,8$ & 0,94 \\
\hline 10 & 2,6 & $-3,5$ & $-0,25$ & 2,6 & $-3,5$ & 2,69 & $-0,9$ & $-0,2$ & $-0,13$ & 0,3 & $-1,3$ & 0,34 \\
\hline 20 & 1,5 & $-3,0$ & $-0,34$ & 1,5 & $-3,0$ & 1,58 & 0,5 & $-2,0$ & 0,51 & 0,0 & $-1,4$ & $-0,04$ \\
\hline 30 & 0,8 & $-2,7$ & $-0,41$ & 0,8 & $-2,7$ & 0,86 & $-0,0$ & $-1,9$ & $-0,07$ & $-0,1$ & $-1,3$ & $-0,14$ \\
\hline 40 & 0,3 & $-2,3$ & $-0,33$ & 0,3 & $-2,3$ & 0,32 & $-0,3$ & $-0,3$ & $-0,30$ & 0,0 & $-0,9$ & $-0,03$ \\
\hline 50 & 0,1 & $-1,9$ & $-0,27$ & 0,1 & $-1,9$ & 0,11 & $-0,0$ & $-1,3$ & $-0,01$ & 0,0 & $-0,8$ & $-0,01$ \\
\hline 60 & 0,1 & $-1,7$ & $-0,21$ & 0,1 & $-1,7$ & 0,15 & 0,0 & $-1,1$ & 0,02 & 0,0 & $-0,6$ & 0,02 \\
\hline
\end{tabular}

Tabla 1. Estadística anuales y totales de temperaturas del terreno medidas en el periodo 2012-2014, en el sondeo situado junto al módulo principal de la base española en la isla Decepción.

Table 1. Annual and total summary of the ground temperatures for the 2012-2014 study period registered at the borehole located in the Spanish Station on Deception Island.

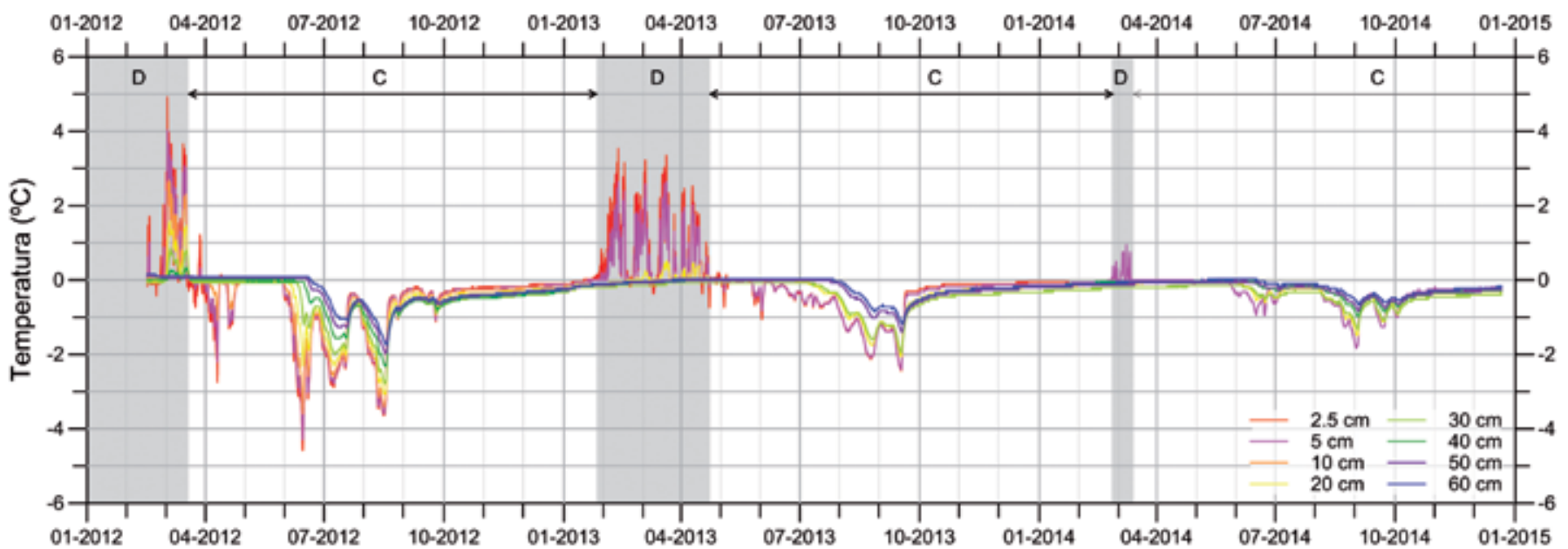

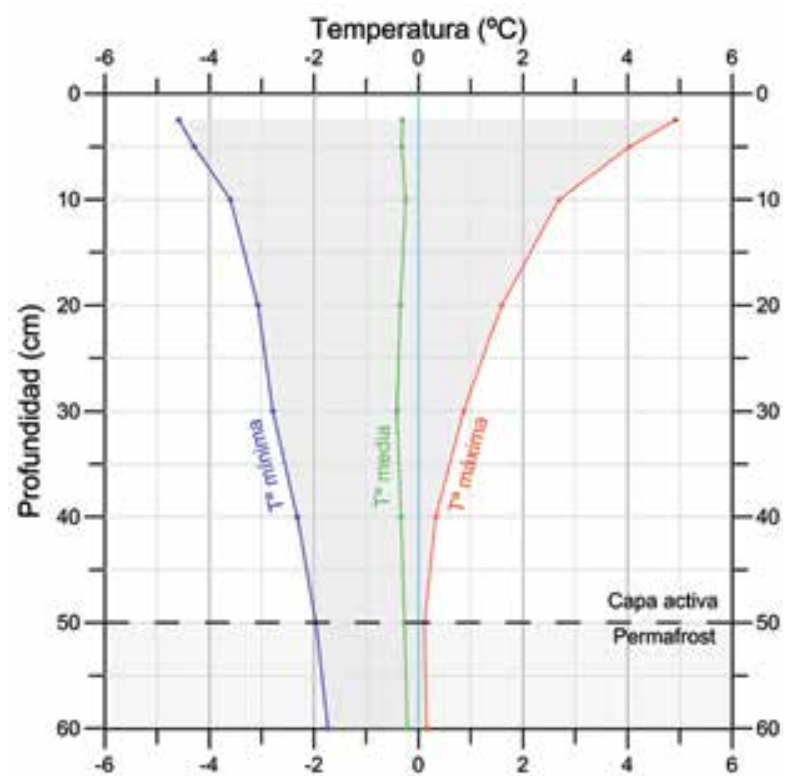

Figura 5. Evolución de las temperaturas del suelo a distintas profundidades entre febrero de 2012 y enero de 2015, a partir de los datos tomados en el sondeo situado junto al módulo principal de la base española en la isla Decepción. Los periodos en los que el suelo permanece congelado (C) y descongelado (D) han ido variando su duración a lo largo del tiempo del estudio.

Figure 5. Evolution of ground temperature measured in a shallow borehole located next to the main module of the Spanish Station on Deception Island. Freezing $(C)$ and thawing $(D)$ periods are marked for each year of the study period (February 2012-January 2015).

Figura 6. Perfil térmico del terreno hasta $60 \mathrm{~cm}$ de profundidad en el entorno de la base española en la isla Decepción, en el que se muestras las temperaturas máximas, mínimas y medias durante del periodo 2012-2014 que permiten determinar el espesor de la capa activa y la inestabilidad del permafrost.

Figure 6. Ground thermal profile at the $70 \mathrm{~cm}$ deep borehole located at the Spanish Station on Deception Island, showing the maximum (red), mean (green) and minimum (blue) temperatures at each depth for the 2012-2014 period, here used to determine the active layer thickness. 
estructura en embudo centrada a temperaturas inferiores a los $0^{\circ} \mathrm{C}$, con una pendiente más elevada en las temperaturas negativas y de tendencia exponencial en las temperaturas positivas. Las temperaturas medias se sitúan muy próximas a los $0^{\circ} \mathrm{C}$ y de forma muy constante a las distintas profundidades.

Este perfil térmico permite establecer que la intersección de las temperaturas máximas con la isoterma de $0^{\circ} \mathrm{C}$ se produce, considerando la precisión de los sensores utilizados, a una profundidad de unos 50 $\mathrm{cm}$. Como se verá a continuación, esto coincide con el espesor de la capa activa medido mediante sondeos mecánicos manuales de monitorización de capa activa en el verano austral de 2012. Así, se establece que la capa activa tiene un espesor de unos $50 \mathrm{~cm}$ de espesor, permaneciendo el terreno permanentemente congelado (permafrost) a partir de dicha profundidad y hasta los $60 \mathrm{~cm}$ de profundidad (profundidad hasta la que se tienen datos térmicos).

\section{Espesor de la capa activa}

La medición en el verano austral de 2012 del espesor del terreno descongelado en el perímetro del módulo principal de la BGdC, así como en los alrededores del mismo, han permitido establecer que en aquel momento el terreno estaba descongelado hasta una profundidad de unos $15 \mathrm{~cm}$ de media, de forma muy uniforme, pero que se incrementa de forma muy importante hacia el frente costero situado delante de la base, alcanzándose medidas de algo más de $90 \mathrm{~cm}$ de profundidad (Figura 7). Sin embargo, esta distribución en el espesor de la capa activa se ve fuertemente modificado en el perímetro del

Figura 7. Mapa de relieves sombreados de la zona de estudio con curvas de nivel (A), con mapa interpolado del espesor de la capa activa, medida en Febrero de 2012, mostrando la tendencia general de aumento del espesor de la capa activa hacia la costa, fuertemente modificada en la localización del módulo principal de la base. Las líneas discontinuas marcan la traza de los perfiles transversales (B) y longitudinales (C) que evidencian el efecto de la base en la tendencia natural del espesor de la capa activa hacia la costa. (Esquema del módulo de la base a diferente escala que el propio perfil del terreno).

Figure 7. Shaded relief map of the study area (A) showing the nearby interpolation of active layer thickness measured in February 2012 (black dots). This map shows the general trend of an increase in the active layer thickness toward the coast, and how this trend is disrupted by the main building of the station with an important increase. Dashed lines mark the location of transverse $(B)$ and longitudinal (C) sections, showing the effect of the station on the natural trend of the active layer thickness toward the coast (The sketch of the station is at different scale to the terrain).
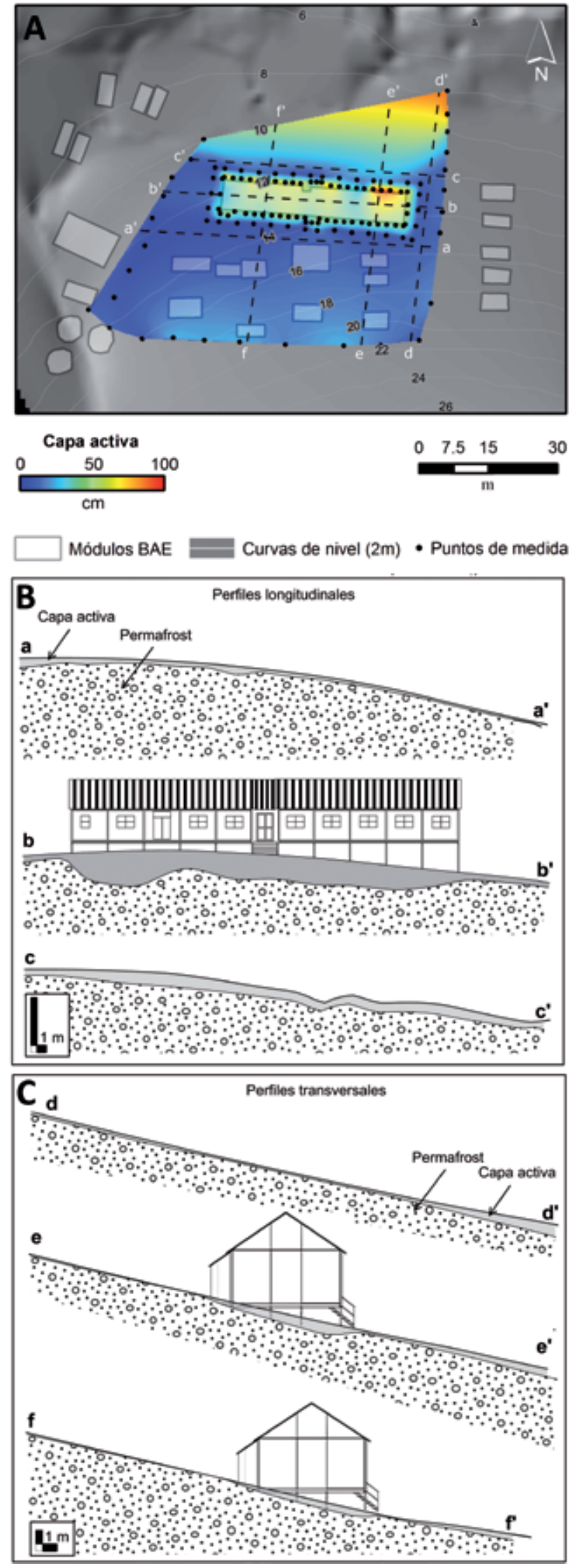


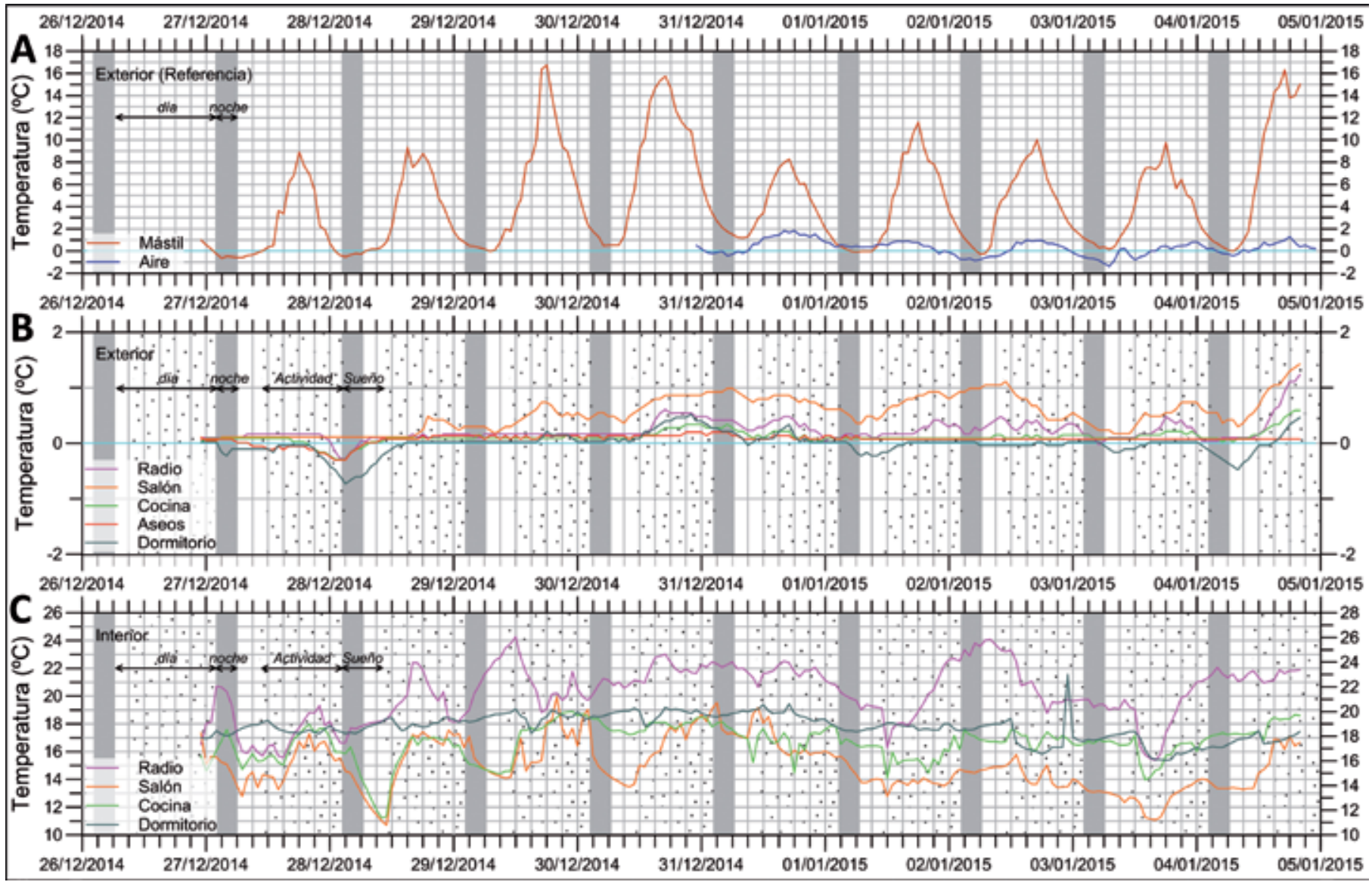

Figura 8. Evolución, para 10 días en la campaña 2014-15, de la temperatura del suelo en el punto de referencia (mástil) y del aire en el exterior de la base situado en un emplazamiento de referencia $(A)$, para el estudio de la evolución de las temperaturas medidas en varios emplazamientos bajo el módulo principal de la base (B), y de las temperaturas en los mismos emplazamientos en el interior (C). Se han marcado los periodos de día y noche (zona en gris) para la latitud y longitud de la base durante las fechas de estudio, así como los periodos de actividad (zona sin punteado) y sueño (zona punteada) del horario de la base.

Figure 8. Air and surface temperature evolution for 10 days in the 2014-2015 field season for a reference site near the main module of the Spanish Antarctic Station and at a nearby reference site $(A)$, and temperature measured at the surface below the station (B) and inside it (C). Plots show night time (grey bands) as well as activity hours in the station (dotted bands).

módulo de vida. Se observa un leve incremento de la capa activa en el perímetro, alcanzándose valores de unos $40-50 \mathrm{~cm}$ de profundidad de suelo descongelado en la parte trasera del módulo, pero de hasta $90 \mathrm{~cm}$ de profundidad en el frente del mismo, especialmente en el ala de vida. Estos valores disminuyen rápidamente a medida que nos alejamos del módulo.

Las variaciones locales del espesor de la capa activa se hacen patentes en los diversos perfiles, transversales y longitudinales a la costa, realizados en la zona de emplazamiento de la base antártica (Figura 8). En ellos se aprecia cómo este espesor aumenta a medida que disminuye la altitud y la distancia al frente costero. Sin embargo, los perfiles realizados a través del módulo principal de la BGdC muestran espesores de capa activa muy elevados y claramente anómalos, respecto a la tendencia que se observa en los otros perfiles del terreno.

\section{Evolución térmica interior/exterior}

El análisis de los datos de temperatura del interior y exterior del módulo principal de la base, medidos en un periodo de 10 días durante la campaña 2013-2014, muestran una evolución muy diferente de la que sigue la temperatura del punto exterior de referencia (Figura 9). La temperatura del suelo en el punto de referencia (junto al mástil de banderas) muestra una clara periodicidad diaria con temperaturas elevadas durante el día, de hasta $17^{\circ} \mathrm{C}$, que descienden hasta los $-1^{\circ} \mathrm{C}$ durante las cortas noches del verano austral. Esta periodicidad está directamente relacionada con 
la evolución de la temperatura del aire en a lo largo de los días de estudio.

En cambio, en los puntos de medida en el exterior bajo el módulo principal de la base se observa que las temperaturas son, en general, muy próximas a los $0^{\circ} \mathrm{C}$, relacionado con la presencia de una capa de hielo y nieve durante el periodo de estudio, así como el apantallamiento de la radiación sobre el suelo bajo la base. En ningún caso se observa una periodicidad similar a la observada en el punto de referencia, sujeto a las condiciones ambientales sin estar apenas cubierto por la nieve salvo el primer día del periodo de estudio. Es de destacar sin embargo, que algunos de los sensores situados bajo la base muestran temperaturas superiores a los $0^{\circ} \mathrm{C}$, especialmente durante el periodo de actividad en la base. El salón o el centro de comunicaciones son los dos habitáculos que alcanzan las temperaturas interiores más elevadas, siendo las temperaturas en el exterior bajo estos emplazamientos también las más elevadas, algo más de $1^{\circ} \mathrm{C}$, observándose un proceso de fusión superficial del suelo y un balance positivo de la energía que intercambia con el exterior. Además, es posible observar cómo las variaciones en la temperatura se relacionan con los periodos de actividad en el interior de la base. Un ejemplo de ello es el ascenso de la temperatura bajo el salón del módulo, especialmente tras la hora de la comida y durante toda la tarde, y descendiendo posteriormente al inicio del periodo de dormir en la base.
Estos incrementos de la temperatura en el exterior, bajo el módulo, están levemente desfasados respecto a los observados en el interior debido a la inercia térmica del suelo. Así, se han registrado temperaturas de hasta $24^{\circ} \mathrm{C}$ en el cuarto de comunicaciones, y nunca inferiores a los $12^{\circ} \mathrm{C}$ en el salón. A diferencia de lo que ocurre en el exterior bajo la base, las mayores temperaturas no se dan en el salón, sino en el centro de comunicaciones, donde se producen numerosos picos térmicos, posiblemente relacionado con incremento de la actividad de los equipos electrónicos. La temperatura es también elevada en los dormitorios, pero mucho más constante, de alrededor de $18^{\circ} \mathrm{C}$; mientras que en la cocina y el salón se producen variaciones de hasta $7^{\circ} \mathrm{C}$ en pocas horas, relacionados con los periodos de actividad en la base los distintos días del estudio.

La comparativa entre temperaturas exteriores bajo el módulo principal de la base con respecto al punto de referencia exterior, es un indicador del grado de protección que ejerce tanto la nieve que se acumula bajo la estructura del edificio como la propia edificación. Por otro lado, la correlación entre las temperaturas del exterior bajo el módulo y las interiores en los mismos emplazamientos sirven como indicador del grado de aislamiento térmico y calefacción generada en el interior del módulo (Figura 9).

También se puede observar cómo comparado con el punto de referencia exterior, las temperaturas bajo el módulo están muy alejadas de una correlación 1:1,

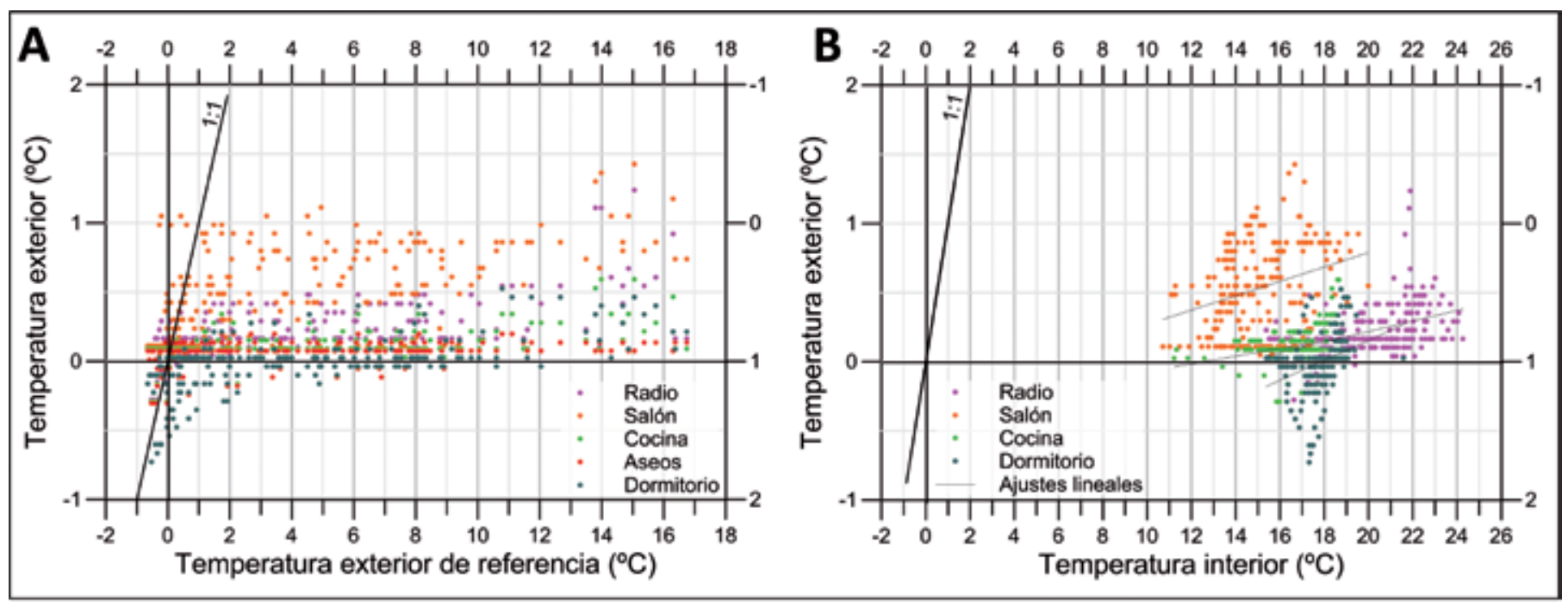

Figura 9. Correlaciones entre datos de temperatura en el exterior y por debajo del módulo principal de la base española en la isla Decepción, respecto al punto de referencia (A), y correlación entre las temperaturas interiores y exteriores para emplazamientos equivalentes de la base (B).

Figure 9. Correlations plots between the temperatures measured at the reference point and below the main module of the Spanish Station on Deception Island $(A)$, and between temperatures measured indoors and outdoors (under the module) on equivalent sites of the module (B). 
de Pablo, M.A., et al., 2017. Análisis del estado de la capa activa en el emplazamiento... Boletín Geológico y Minero, 128 (1): $69-92$

\begin{tabular}{|l|l|c|c|c|c|}
\hline \multicolumn{2}{|c|}{ Punto de medida } & $\begin{array}{c}\text { Máx. } \\
\left({ }^{\circ} \mathbf{C}\right)\end{array}$ & $\begin{array}{c}\text { Mín. } \\
\left({ }^{\circ} \mathbf{C}\right)\end{array}$ & $\begin{array}{c}\text { Media } \\
\left({ }^{\circ} \mathbf{C}\right)\end{array}$ & $\begin{array}{c}\text { Índice de calor } \\
\left({ }^{\circ} \mathbf{C}\right)\end{array}$ \\
\hline \multirow{4}{*}{ Interior } & Radio & 24,2 & 15,4 & 20,21 & $4.324,6 \pm 5$ \\
\cline { 2 - 6 } & Salón & 20,0 & 10,7 & 15,03 & $3.215,5 \pm 5$ \\
\cline { 2 - 6 } & Cocina & 18,9 & 11,2 & 16,62 & $3.555,9 \pm 5$ \\
\cline { 2 - 6 } & Dormitorios & 21,6 & 15,4 & 17,75 & $3.797,8 \pm 5$ \\
\hline \multirow{4}{*}{ Exterior } & Radio & 1,2 & $-0,3$ & 0,23 & $49,3 \pm 5$ \\
\cline { 2 - 6 } & Salón & 1,4 & 0,05 & 0,53 & $113,5 \pm 5$ \\
\cline { 2 - 6 } & Cocina & 0,6 & $-0,3$ & 0,11 & $26,1 \pm 5$ \\
\cline { 2 - 6 } & Aseos & 0,2 & $-0,3$ & 0,07 & $18,1 \pm 5$ \\
\cline { 2 - 6 } & Dormitorios & 0,5 & $-0,7$ & $-0,01$ & $12,5 \pm 5$ \\
\hline \multirow{3}{*}{ Referencia } & & 16,7 & $-0,7$ & 4,46 & $961,2 \pm 5$ \\
\hline
\end{tabular}

Tabla 2. Estadísticas básicas de los datos de temperatura medidos en el interior y exterior del módulo principal de la base española en la isla Decepción, así como en el emplazamiento de referencia, e índices de calentamiento calculador para el periodo de estudio.

Table 2. Summary of the temperature data (inside and outside) and heating indexes for the study period at the main building of the Spanish Station on Deception Island, as well as at the reference site.

siendo en general todas las temperaturas muy próximas a cero en el exterior (Figura 9). Debido a que el punto de referencia se encuentra expuesto al calentamiento por radiación solar en un terreno prácticamente libre de hielo y nieve, se alcanzaron, en ese punto, temperaturas de hasta $17^{\circ} \mathrm{C}$.

Los índices de calentamiento $\left({ }^{\circ} \mathrm{C}\right)$ calculados en el interior y el exterior del módulo de vida (Tabla 2; Figura 10) permiten establecer para el exterior, que los puntos donde se alcanzan mayores temperaturas son el salón, el centro de comunicaciones, la cocina y los dormitorios; alcanzándose valores máximos de menos de $115^{\circ} \mathrm{C}$ para el periodo de estudio (10 días), con una media de $11,5^{\circ} \mathrm{C} /$ día. Sin embargo, los índices de calor calculados para los datos del interior disminuyen desde los $4.750 \pm 5^{\circ} \mathrm{C}$ en el centro de comunicaciones, a los casi $3.250 \pm 5^{\circ} \mathrm{C}$ en el salón, resultando en medias de entre $475 \pm 5^{\circ} \mathrm{C} /$ día y $325 \pm 5^{\circ} \mathrm{C} /$ día, respectivamente. El valor de índice de calor calculado para el punto de medida exterior de referencia es de $961 \pm 5^{\circ} \mathrm{C}$ para el periodo de estudio de 10 días.

\section{Discusión}

\section{La capa activa y su afección por la base}

Los datos de temperatura del terreno, medidos en el sondeo ubicado en la proximidad de la base, obtenidos entre febrero de 2012 y enero de 2015, muestran que éste se congela durante el invierno, como sugieren las condiciones ambientales existentes. Estas

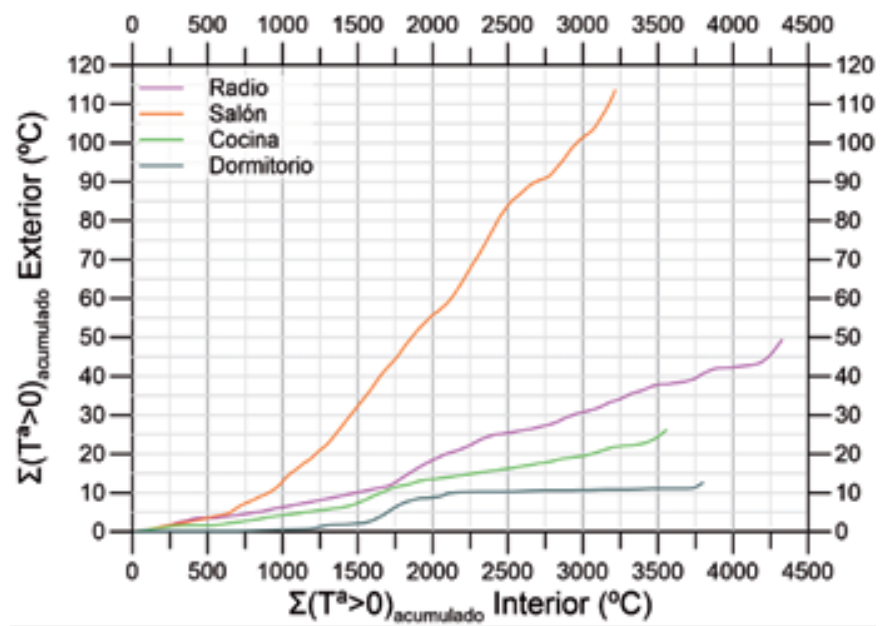

Figura 10. Correlación entre los índices de calentamiento calculados para el interior y el exterior de distintos emplazamientos de la base española en la isla Decepción.

Figure 10. Heating indexes outside/inside correlation plot derived from temperature data measured for a 10-day period in the Spanish Station on Deception Island.

condiciones climáticas permiten la existencia de una capa activa durante los periodos estivales, y la presencia de permafrost a partir de los $50 \mathrm{~cm}$ de profundidad. Estos resultados son coherentes con los obtenidos en la zona de Crater Lake, a menos de $1 \mathrm{~km}$ de distancia en dirección sur de la base, y a unos $80 \mathrm{~m}$ de altitud (ej., Ramos et al., 2010, 2012; Vieira et al., 2010). Las diferencias en el espesor de la capa activa 
de Pablo, M.A., et al., 2017. Análisis del estado de la capa activa en el emplazamiento... Boletín Geológico y Minero, 128 (1): $69-92$

en ambos emplazamientos, situados en zonas de similar pendiente y orientación y con materiales geológicos similares, se deben a la variación de altitud.

Por el valor de la temperatura media del sondeo situado junto a la base y tomadas de forma continua a lo largo de los últimos años (de unos $-0.5^{\circ} \mathrm{C}$; Tabla 1; Figura 6), podemos establecer que el permafrost existente, al menos en su parte superior, es de carácter inestable. Esto lo hace fácilmente degradable en caso de ascenso de la temperatura (ya sea de forma natural o antrópica). Estos resultados son además congruentes con los obtenidos de los sondeos mecánicos manuales llevados a cabo en febrero de 2012, apreciándose para el mismo lugar donde se encuentra el sondeo térmico un espesor de la capa activa de $47 \mathrm{~cm}$ (Figura 6).

De esta forma, podemos suponer que las medidas de los sondeos mecánicos manuales realizados en ese año son representativos del estado térmico de la capa activa medida en todo el entorno de la BGdC en el año 2012. Sin embargo, no podemos descartar que por efecto de las temperaturas interiores de la base las medidas del espesor de la capa activa no hayan aumentado entre 2012 y 2015 . En todo caso, partiendo de esta situación como marco de referencia para el presente trabajo, podemos establecer que la capa activa en la zona de emplazamiento de la base aumenta a medida que se reduce la distancia a la costa y se disminuye en cota, como es esperable debido a que en estas zonas las temperaturas son mayores por el atemperamiento del mar y la reducción de la altitud. Las medidas de espesor de capa activa tomadas más cerca del frente de costero alcanzan valores de casi $90 \mathrm{~cm}$, indicando un rápido crecimiento de ésta y la consiguiente desaparición del permafrost.

La mayor duración del periodo de congelación del terreno (Figura 5) ha sido interpretada aquí, como un efecto de la mayor duración del periodo de nieves. Situaciones similares han sido observadas con carácter regional, tanto en la zona de Crater Lake en la propia isla Decepción, como también en las penínsulas Hurd y Byers de la isla Livingston (Jiménez, 2013; de Pablo et al., 2014, 2016).

Frente a este marco de referencia, las medidas realizadas en todo el perímetro del módulo principal de la BGdC muestran un incremento muy importante de la capa activa, alcanzándose valores de hasta $90 \mathrm{~cm}$ en la fachada frontal (orientada al norte $y$, por tanto, para esas latitudes, la que mayor tasa de insolación tiene). Este incremento es especialmente importante también en el extremo este, asociado al ala más antigua de la base, y a los lugares en cuyo interior las temperaturas son más altas. Estas últimas se encuentran asociadas a la habitación donde se concentran los equipos electrónicos de comunicaciones (radio, telefonía, internet, satélite, etc.), el salón donde se concentran las actividades comunes de vida (comedor, área de descanso, zona de conferencia y proyecciones, etc.), y la cocina. Es de destacar que el espesor de la capa activa sólo se incrementa de forma leve en la fachada posterior (orientada al sur y, por tanto, con menos horas de insolación), y con valores muy similares a lo largo de toda ella, independientemente de si se trata del ala de vida o de dormitorios (Figura 7).

Este escaso incremento del espesor de la capa activa en la fachada posterior, y su uniformidad a lo largo de toda ella, nos hace pensar en que el efecto del edificio de la propia base es el causante del mismo. Esto podría ser causado, bien por efecto pantalla frente a los vientos y la zona de acumulación de nieve. Un efecto que el edificio tiene sobre el terreno es el de protegerlo en gran medida de las condiciones ambientales. El edificio sólo está al nivel del terreno en $3 / 4$ partes de la fachada posterior, quedando elevado en el resto del perímetro (por estructuras metálicas apoyadas en el terreno mediante dados de hormigón o tacos de madera). Esta configuración protege en cierta medida al terreno de la intemperie, tanto del calentamiento por radiación solar durante el verano, como del enfriamiento durante el invierno, teniendo un efecto de aislante térmico, al igual que ocurre con la capa de nieve (ej., Zhang, 2005). Por otro lado, esta estructura del edificio hace que se puedan acumular bajo él importantes cantidades de nieve, tal y como se ha podido comprobar a lo largo de diversas campañas, con su consiguiente efecto aislante.

Sin embargo, las variaciones observadas en el espesor de la capa activa en el perímetro de la fachada frontal pueden estar relacionadas en cierta medida con esta misma causa, pero otra u otras deben superponerse dado que los espesores de capa activa medidos son muy diversos a lo largo de toda la fachada. Estas otras causas podrían estar por tanto controlando dicha variabilidad, incluyendo diferencias de temperatura en el interior, diferentes elevaciones de la base respecto al terreno, diferente facilidad para la aireación del hueco entre la base y el terreno, diferencias constructivas y aislantes de los módulos, o diferentes periodos de exposición (es decir, diferente tiempo desde que las estructuras fueron levantadas en la zona).

La acumulación de nieve en el entorno de los módulos, mucho mayor que la existente en el terreno circundante observada al inicio de algunas campañas, sugieren que los propios módulos tienen un efecto pantalla que hace que se generen vórtices de corriente que acumula la nieve entre los distintos módulos y 
de Pablo, M.A., et al., 2017. Análisis del estado de la capa activa en el emplazamiento... Boletín Geológico y Minero, 128 (1): $69-92$

cerca de los mismos, no permitiendo el paso del viento. De hecho, esto ya ha sido estudiado en profundidad para el diseño de las últimas bases antárticas, y valga como ejemplo el diseño de la base coreana Jang Bogo, situada en la bahía Terra Nova $\left(74^{\circ} 37^{\prime} \mathrm{S}\right.$, $\left.164^{\circ} 12^{\prime} \mathrm{E}\right)$, en el Mar de Ross. En esta base, para facilitar la aireación del piso del módulo y evitar la acumulación de nieve en los alrededores, se han construido módulos elevados sobre pilares y con diseños más aerodinámicos y de aristas truncadas, para dejar pasar los vientos dominantes (Ropero, 2013).

Aunque la excesiva acumulación de nieve entre los módulos de la BGdC es un hecho, los datos disponibles en este trabajo no nos permiten discernir a priori cuál de las causas anteriormente citadas pueden estar presentes en la zona. Sin embargo, las observaciones de los datos de temperatura en el interior y el exterior del módulo principal de la base apuntan a que algunas de ellas tienen gran importancia, como es el caso de la transmisión de calor por efecto de la radiación térmica desde el interior con el consiguiente calentamiento del terreno. Del estudio de las temperaturas en el interior y el exterior (Figura 9) se observa un incremento de la temperatura del terreno con un desfase de aproximadamente un par de horas, y todo ello a pesar de la existencia de una capa de hielo y nieve (al menos durante los primeros días del estudio en la campaña 2014-2015). Este efecto implica un sistema de aislamiento del suelo de la base, sin duda, poco eficiente. Las correlaciones (o la falta de ellas) entre los datos de temperatura en el interior y el exterior de la base corroboran que, si bien la transmisividad no es directa, como cabría esperar si la base no tuviera cerramientos, si se observa que las temperaturas exteriores están levemente influenciadas por las del interior (Figura 9).

Por otro lado, los datos térmicos parecen mostrar una cierta incongruencia. En el interior del módulo de vida las temperaturas más altas se alcanzan en el cuarto de comunicaciones (Figura 9), pero en el exterior bajo la base se dan asociadas al salón. La ubicación del cuarto de comunicaciones en la esquina del edificio permite que este sector tenga una mayor aireación y pérdidas laterales de calor limitando el calentamiento del suelo en esta zona.

La absorción de energía es también mayor en el ala de vida que en el de dormitorios del módulo principal de la base. Esto podría estar relacionado con dos factores: una mayor separación entre el terreno y la estructura de la base en el ala de dormitorios, permitiendo una mayor aireación bajo la misma y una fuerte disipación del calor que se genera en el interior y se transmite al exterior; y/o un menor tiempo de interacción entre el suelo y la estructura, ya que este ala de dormitorios fue levantado en la campaña 2009-2010, y, aunque no hemos podido comprobar para este estudio las capacidades aislantes, empleando materiales diferentes para el piso y estructura de este ala. Esto puede explicar la asimetría en el espesor de la capa activa observado en la fachada frontal del módulo. Sin embargo es necesario recalcar que, a pesar de esta diferencia, frente a la fachada norte del ala de dormitorios la capa activa medida en 2012 alcanza valores de hasta $70 \mathrm{~cm}$, por lo que a pesar de su bajo periodo de exposición y de una mayor elevación respecto al terreno, también se está produciendo una mayor descongelación del mismo que la esperada en comparación con los valores del área circundante que alcanzan profundidades de la capa activa del orden de unos 10 a $15 \mathrm{~cm}$.

Por todo ello, a pesar de lo limitados de los datos disponibles, y aunque no es posible discernir el efecto de otras causas en el área de la mayor descongelación del suelo bajo el módulo principal de la base, los datos revelan que las temperaturas elevadas del interior del módulo están siendo transmitidas al exterior en mayor o menor medida, causando una aceleración en la descongelación del suelo, y que ésta ocurre de forma diferente en cada punto bajo la base.

\section{Implicaciones y problemas asociados}

La descongelación estacional de los suelos (capa activa) de forma uniforme bajo ella no debería tener, a priori, grandes implicaciones directas en las infraestructuras de la base antártica como asientos diferenciales. Sin embargo, la formación de una capa activa de mayor espesor en el módulo principal de la BGdC, sí que tiene una serie de implicaciones directas sobre el módulo, y otras indirectas que llegarán a afectar a éste y a otras infraestructuras de la base (como conductos y depósitos enterrados), o al avance de la erosión del frente costero (Figura 11) (Recio, 2015).

En primer lugar, un mayor espesor de la capa activa supone una reducción de la resistencia mecánica del suelo, ya que éste está formado por materiales sueltos y de bajo grado de consolidación en su parte superior (Goyanes, 2015; LIE-Ropero, 2015). Al estar asentada la estructura sobre estos materiales, la variabilidad espacial de la degradación del espesor de la capa activa como consecuencia del impacto térmico de la base tendría gran incidencia al tratarse de asientos diferenciales debido a la distribución tan irregular de la profundidad de la capa activa bajo la base.

Aunque esto fue una de las motivaciones para la realización de este trabajo en 2012, en la actualidad se sabe que los problemas detectados en el piso del ala 
de vida del módulo principal no se deben a asientos diferenciales, sino a problemas de dilataciones y humedades, o a la degradación de los materiales constituyentes con el paso del tiempo. De hecho, aunque no se han detectados grandes asientos en la estructura de este módulo, ya que dispone de pocos grados de libertad para presentar movimientos, si estos se produjesen la estructura se movería como un todo y aunque algún apoyo quedara al aire, la estructura tiene capacidad para absorber esta carencia presentando cierta capacidad de deformación y trabajando como una viga empotrada. En todo caso, no se puede perder de vista que este proceso de asientos (totales y diferenciales) sí podría suponer problemas para otros de los módulos de la BGdC.

Por otro lado, la pérdida del hielo que ejerce como ligante de los piroclastos que forman el terreno en esta zona, supone que éstos pierdan su consolidación y aumentan su grado de erosionabilidad. De esta forma, quedan disponibles para ser arrastrados por la escorrentía superficial y la arroyada, ya sea concentrada o en lámina. Esta pérdida de materiales, de producirse, podría llegar a generar el descalce de algunos de los puntos de apoyo de los módulos de la $\mathrm{BGdC}$, con los consiguientes problemas estructurales. Pero este tipo de procesos, no relacionados con el calentamiento del terreno por actividad antrópica directa, se ha observado en otros módulos de la base, como los de náutica y almacén situados al oeste del módulo principal.

Esta pérdida de consolidación, así como el aumento o la concentración del agua de escorrentía superficial, pudieron ser durante el inicio de la campaña 2014-2015 los responsables de la enorme aceleración de los procesos de erosión por escorrentía que sobrexcavaron las cárcavas existentes en la ladera entre la base y el frente costero (Figura 11), y excavó otras nuevas de hasta 1,5 metros de profundidad (Recio, 2015). Éstas dejaron al descubierto algunas infraestructuras clave de la base, como son los depósitos de depuración de las aguas grises y negras del módulo principal, derribaron una antena de comunicaciones, y arrastraron al frente costero gran cantidad de materiales.

Este aumento de la erosión por excavación de cárcavas y regueros, además de la modificación del paisaje natural y de las consiguientes dificultades para el tránsito de personal y vehículos en el entorno de la base, supone una forma de dejar expuesto al aire zonas del terreno situadas a mayor profundidad. Este proceso, a su vez, contribuiría a la descongelación del permafrost remanente en la zona, acrecentando de nuevo, en un proceso de retroalimentación positiva, la sensibilidad a la erosión del terreno.
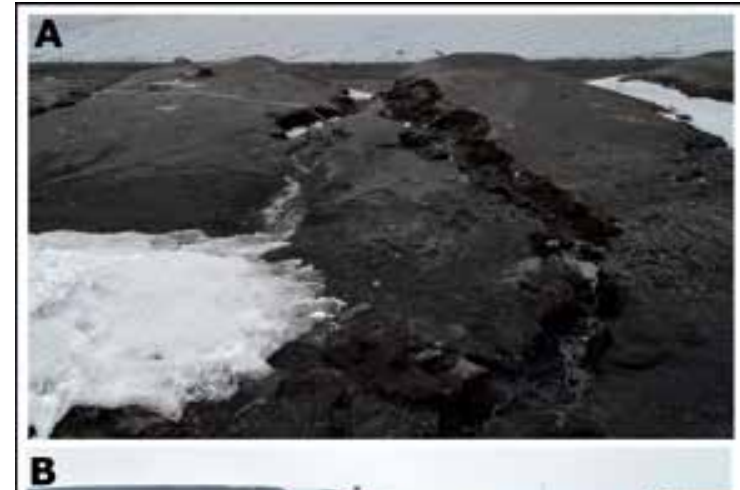

B
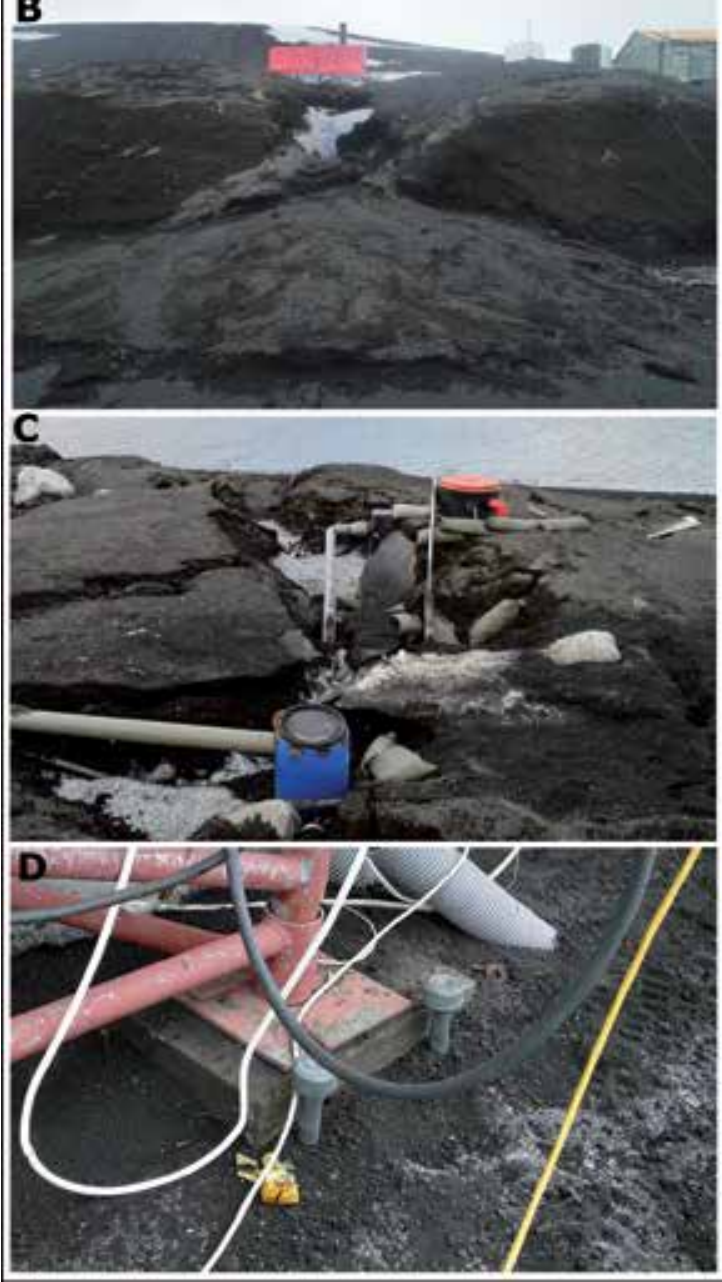

Figura 11. Ejemplos de los procesos erosivos en el entorno de la base Gabriel de Castilla y sus efectos: (A) excavación de nuevas cárcavas; (B) pérdida de material que se acumula temporalmente en la zona de playa; (C) desperfectos en las infraestructuras enterradas de la base (fosas sépticas y canalizaciones); (D) posible asientos diferenciales en los apoyos de la base, y pérdida de materiales finos por lavado, que cambia las propiedades geotécnicas e hidrogeológicas del terreno.

Figure 11. Pictures of some examples of the erosion effects on the "Gabriel de Castilla" Station site: (A) gully excavation; (B) eroded material accumulated on the beach; $(C)$ damage to the buried infrastructure (septic tanks and pipes); and (D) loss of fine grained material by washing, changing the geotechnical and hydrogeological terrain properties. 
de Pablo, M.A., et al., 2017. Análisis del estado de la capa activa en el emplazamiento... Boletín Geológico y Minero, 128 (1): $69-92$

\section{Actuaciones de control y necesidades de investiga- ción futura}

Los resultados obtenidos han evidenciado que tanto la base como la actividad en el interior de ella están causando un incremento en la fusión de la capa activa en su área de influencia, haciendo que ésta tenga un mayor espesor, esto es, causando la degradación del permafrost en la zona. Por ello, es necesario tomar una serie de medidas dirigidas a reducir las causas del aumento de dicho espesor de la capa activa, y por otro, reducir los efectos de la misma. El control de la temperatura interior, el aumento del aislamiento bajo la base y facilitar la aireación bajo la base son algunas de las actuaciones que deberían considerarse para las siguientes campañas. Estas actuaciones permitirían, no sólo reducir las causas y los efectos de la fusión del permafrost y la capa activa, sino que conllevarían una mejora de la eficiencia energética de la base, una reducción del consumo de combustible (energía), y la consiguiente reducción de la huella de carbono causada por la propia base, la primera base antártica con certificación de sostenibilidad ambiental ISO 14001.

A estas acciones podrían sumarse otras de carácter ingenieril más agresivas (Recio, 2015), que podrían conducir a un rediseño de los módulos en el caso de futuras ampliaciones o remodelaciones de la base; considerando, no solo el aislamiento térmico, sino la reducción de su influencia en factores como el viento, para reducir también la acumulación excesiva de nieve junto a los módulos (Ropero, 2013).

Debido a la importancia que el régimen térmico del terreno tiene en las condiciones de estabilidad de la base y del terreno natural en la zona de estudio, tal y como ha quedado patente a lo largo de este trabajo, es necesario continuar el estudio y monitorización de la capa activa y el permafrost en el entorno de la BGdC, cumpliendo así con una de las recomendaciones del informe resultante de la inspección del Tratado Antártico realizada a la base el 31 de diciembre de 2014 (ATS, 2015).

Para continuar estas actuaciones, es necesario continuar la monitorización térmica de la capa activa y el techo del permafrost en el sondeo situado junto al módulo principal de la base, incluyendo la realización de otros sondeos para establecer el estado térmico del terreno, especialmente en el módulo de vida y el módulo científico; la medición sistemática y anual del espesor de la capa activa en la zona de la base; o la realización de ensayos geofísicos no destructivos (técnicas de sísmica de refracción y de tomografía eléctrica) que permitan la verificación de los resultados arrojados por los sondeos térmicos y las medidas de espesor de la capa activa, así como la modelización del terreno, entre otros estudios.

\section{Conclusiones}

El análisis de los resultados obtenidos en el estudio de la capa activa y el permafrost en relación al módulo principal de la Base Antártica Española Gabriel de Castilla, realizado entre febrero de 2012 y enero de 2015, pueden sintetizarse de la siguiente manera:

- El espesor de la capa activa derivada de los datos térmicos de un sondeo situado en la fachada posterior (de orientación sur) del módulo principal de la base es de unos $50 \mathrm{~cm}$.

- La temperatura media de los primeros $60 \mathrm{~cm}$ del terreno en el sondeo es de unos $-0.5^{\circ} \mathrm{C}$, lo que hace que el permafrost que se sitúa inmediatamente bajo la capa activa tenga un carácter inestable, pudiendo degradarse muy fácilmente ante mínimas variaciones de las condiciones ambientales.

- La evolución de los datos térmicos en el periodo de estudio revelan un incremento del periodo en el que el terreno permanece congelado por reducción del tiempo que el terreno permanece libre de hielo y nieve, al igual que se ha observado en otros emplazamientos de las islas Shetland del Sur.

- Las medidas de la capa activa en febrero de 2012 en el entorno de la base muestran que el espesor medio es de unos $15 \mathrm{~cm}$, incrementándose rápidamente hacia el escarpe del frente de costa.

- Las medidas del espesor de la capa activa aumentan drásticamente en el perímetro del módulo principal y muestran que éste tiene un valor medio de unos $45 \mathrm{~cm}$, llegando a alcanzar los $90 \mathrm{~cm}$ de espesor en la fachada frontal (de orientación norte) en el ala del módulo de vida.

- Las medidas de temperatura en el interior y el exterior de la base, realizadas entre diciembre de 2014 y enero de 2015, han mostrado que el terreno bajo la base no tiene una respuesta similar a la del terreno natural, debido el efecto pantalla que ejerce la propia edificación.

- El terreno, a pesar de no estar en contacto directo con la edificación, responde térmicamente a las variaciones de temperatura en el interior, y de forma diferente en función de las temperaturas internas en cada una de las dependencias, y de la facilidad de aireación bajo la Base.

Con todos estos datos, en este trabajo se concluye que el módulo principal de la base tiene un efecto térmico en el comportamiento del permafrost y el espesor de la capa activa, contribuyendo a su degradación. 
de Pablo, M.A., et al., 2017. Análisis del estado de la capa activa en el emplazamiento... Boletín Geológico y Minero, 128 (1): $69-92$

Desde un punto de vista termodinámico, el módulo cuando se encuentra operativo se puede modelizar como una fuente emisora de calor al terreno por el efecto combinado de diferentes procesos de transmisión térmica (conducción, convección y radiación); ya que es insuficiente el aislamiento térmico que tiene en la actualidad. Todo ello altera directamente la temperatura del terreno bajo las edificaciones. Esta influencia es irregular en los distintos puntos del terreno bajo la base, dando lugar a un espesor variable de la capa activa.

Se apunta la importancia que puede alcanzar este proceso de continuar afectando, no sólo a un proceso natural que no debiera verse alterado por la actividad antrópica en un entorno protegido como es la Antártida, sino también a la estabilidad de la propia edificación de forma directa e indirecta. El bulbo de presiones bajo el módulo se vería modificado directamente, produciendo entre otros efectos, asientos diferenciales y descalces de los cimientos; pudiendo poner en serio riesgo la estabilidad estructural del módulo principal a largo plazo. De forma indirecta, se produce la aceleración de los procesos de erosión del terreno por incremento del agua de escorrentía y la reducción de la cohesión de los materiales volcánicos, ya poco cohesionados, sobre los que se asienta la base.

Ante estos primeros resultados, resulta conveniente continuar la labor de investigación de forma más detallada y sistemática, tanto del módulo principal de la base, como del módulo científico (más antiguo que éste), y de los módulos auxiliares para establecer la situación real del permafrost en el emplazamiento y que sirva de base para detectar futuros problemas relacionados con la fusión del permafrost.

\section{Agradecimientos}

El presente trabajo ha sido realizado gracias al apoyo de los diferentes jefes y dotaciones de la Base Antártica Española Gabriel de Castilla durante las campañas de 2008-2009 a 2014-2015, pertenecientes al Ejército de Tierra del Ministerio de Defensa del Gobierno de España. Nuestro especial agradecimiento al Comandante Teniente coronel Antonio Casals, jefe de base durante la campaña 2011-2012, quien tuvo la iniciativa y realizó el primer contacto con este equipo de investigación, y al Brigada Adrián Antonio Petit, de la dotación de la base durante la citada campaña, por su inestimable ayuda con la perforación del sondeo térmico realizado junto a la base. Así mismo, deseamos agradecer a las dotaciones de los buques de investigación oceanográfica (BIOs) "Las Palmas" y "Hespérides" por facilitar el acceso a la isla Decepción (Antártida) durante las diferentes campañas. Finalmente, deseamos agradecer las facilidades por parte del Comité Polar Español y el Gestor del Programa Polar del Ministerio de Economía y Competitividad para el desarrollo de estas investigaciones.

Este trabajo ha sido parcialmente posible gracias a diferentes proyectos de investigación sobre los suelos congelados de las islas Shetland del Sur: PERMAPLANET (CTM2009-10165-E), ANTARPERMA (CTM2011-15565-E), PERMATHERMAL, Y PERMASNOW (CTM2014-52021-R), del Plan Nacional de I+D.

Los autores quieren agradecer a Dr. Jerónimo López-Martínez y Dr. Juan José Durán su detallada revisión del manuscrito y sus comentarios para mejorar el presente trabajo.

\section{Referencias}

Almendros, J., Carmona, E. e Ibáñez, J. 2004. Precise determination of the relative wave propagation parameters of similar events using a small-aperture seismic array. Journal of Geophysical Research, 109: B11308, doi:10.1029/2003JB002930.

Andersland, O.B. y Ladanyi, B. 1994. An Introduction to Frozen Ground Engineering. Chapman and Hall, New York. 352 pp.

Anisimov, O.A., Shiklomanov., N.I. y Nelson, F.E. 1997. Global warming and active layer thickness: results from transient general circulation models. Global and Planetary Change, 15, 61-67.

ATS, 1959. The Antarctic Treaty (December 1st, 1959. Washington D.C., USA). Antarctic Treaty Secretariat, Argentina. [www.ats.aq/e/ats.htm]

ATS, 1991. The protocol on the environmental protection to the Antarctic Treaty (October 4th, 1991. Madrid, Spain). Antarctic Treaty Secretariat, Argentina. [www.ats.aq/e/ ep.htm]

ATS, 2012. Zona Antártica Especialmente Protegida No ${ }^{\circ} 140$, (Partes de la isla Decepción): Plan de Gestión revisado. Antarctic Treaty Secretariat, Argentina. [www.ats.aq/

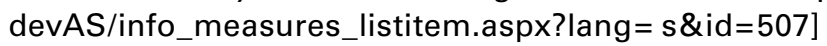

ATS, 2015. Antarctic Treaty inspections program report 2014-15: Report of the Antarctic Treaty Inspections undertaken jointly by the United Kingdom and the Czech Republic in accordance with Article VII of the Antarctic Treaty and Article 14 of the Environmental Protocol. Antarctic Treaty Secretariat, Argentina. [www.ats.aq/

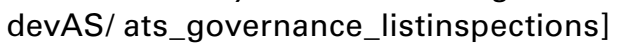

Baker, P., McReath, M.A., Harvey, M.R., Roobol, M.J. y Davies, T.G. 1975. The geology of the South Shetland Islands: V. Volcanic evolution of Deception Island. British Antarctic Survey Scientific Reports, 78, 107 pp. 
de Pablo, M.A., et al., 2017. Análisis del estado de la capa activa en el emplazamiento... Boletín Geológico y Minero, 128 (1): $69-92$

Bañón, M. y Vasallo, F. 2015. AEMET en la Antártida: Climatología y meteorología sinóptica en las estaciones meteorológicas españolas en la Antártida. Agencia Estatal de Meteorología, Madrid. 152 pp.

Bromwich, D.H., Nicolas, J.P., Monaghan, A.J., Lazzara, M.A., Keller, L.M., Weidner, G.A. y Wilson. A.B. 2013. Central West Antarctica among the most rapidly warming regions on Earth. Nature Geoscience, 6(2), 139-145.

Brown, J., Nelson, F.E. y Hinkel, K.M. 2000. The circumpolar active layer monitoring (CALM) program research designs and initial results. Polar Geography, 3, 165-258.

CSA, 2014. Thermosyphon foundations for buildings in permafrost regions. Standards council of Canada, Canada. CAN/CSA-S500-14. 44 pp.

Danby, R.K. y Hik, D.S. 2007. Responses of white spruce (Picea glauca) to experimental warming at a subarctic alpine treeline. Global Change Biology, 13(2), 437-451.

de Pablo, M.A., Ramos, M. y Molina, A. 2014. Thermal characterization of the active layer at the Limnopolar Lake CALM-S site on Byers Peninsula (Livingston Island), Antarctica. Solid Earth, 5, 721-739.

de Pablo, M.A., Molina, A. y Ramos, M. 2016. Snow cover evolution at the Limnopolar Lake CALM site on Byers peninsula, Livingston Island, Antarctica, on 2009-2014. Catena (en revisión).

French , H.M. 2007. The Periglacial Environment. John Wiley \& sons, Ltd. 480 pp, England.

Gadek, B. y Leszkiewicz, J. 2010. Influence of snow cover on ground surface temperature in the zone of sporadic permafrost, Tatra Mountains, Poland and Slovakia. Cold Regions Science and Technology, 60, 205-211.

González-Casado, J.M., Giner-Robles, J.L. y LópezMartínez, J. 2000. Bransfield Basin, Antarctic Peninsula: Not a normal backarc basin. Geology, 28, 1043-1046.

Goyanes, G. 2015. Control climático e interacciones permafrost-volcanismo, Isla Decepción, Antártida. Tesis Doctoral, Departamento de Ciencias Geológicas, Facultad de Ciencias Exactas y Naturales, Universidad de Buenos Aires, 317 pp.

Goyanes, G., Vieira, G., Caselli, A., Cardoso, M., Marmy, A., Santos, F., Bernardo, I. y Hauck, C. 2014a. Local influences of geothermal anomalies on permafrost distribution in an active volcanic island (Deception Island, Antarctica). Geomorphology, 225, 57-68.

Goyanes, G., Vieira, G., Caselli, A., Mora, C., Ramos, M., de Pablo, M.A., Neves, M., Santos, F., Bernardo, I., Gilichinsky, D., Abramov, A., Batista, V., Melo, R., Nieuwendam, A., Ferreira, A. y Oliva, M. 2014b. Régimen térmico y variabilidad espacial de la capa activa en isla Decepción, Antártida. Revista de la Asociación Geológica Argentina, 71(1), 112-124.

Grad, M., Guterch, A. y Sroda, P. 1992. Upper crustal structure of Deception Island area, Bransfield Strait, West Antarctica. Antarctic Science, 4(4), 469-476.

Gubler, S., Fiddes, J., Keller, M. y Gruber, S. 2011. Scaledependent measurement and analysis of ground surface temperature variability in alpine terrain. The Chryosphere, 5, 431-443.
Guglielmin, M., Dalle Fratte, M. y Cannone, N. 2014. Permafrost warming and vegetation changes in continental Antarctica. Environmental Research Letters, 9. 045001. doi:10.1088/1748-9326/9/4/045001.

Guglielmin, M. y Vieira, G. 2014. Permafrost and periglacial research in Antarctica: New results and perspectives. Geomorphology, 225, 1-3.

Harris, S.A., French, H.M., Heginbottom, J.A., Johnston, G.H., Ladanyi, B., Sego, D.C. y van Everdingen R.O. 1988. Glossary of permafrost and related ground-ice terms. Permafrost Subcommittee. Associate Committee on Geotechnical Research, National Research Council of Canada, $154 \mathrm{pp}$.

Hauck, C., Vieira, G., Gruber, S., Blanco,J.J.y Ramos, M. 2007. Geophysical identification of permafrost in Livingston Island, maritime Antarctica. Journal of Geophysical Research, 112, F02S19, doi: 10.1029/2006JF000544.

Hawkes, D. 1962. The structure of the Scotia Arc. Geological Magazine, 99, 85-91.

Holubec, I. 2008. Flat loop thermosyphon foundations in warm permafrost. I. Holubec Consulting Inc. Canadá. 119 pp.

Ibañez, J., Almendros, J., Carmona, E., Martínez-Arévalo, C. y Abril, M. 2003. The recent seismovolcanic activity at Deception Island volcano. Deep-Sea Research II, 50: 1611-1629.

Jiménez, J.J. 2013. Estudio de la variabilidad de la capa activa en el entorno de la BAE Juan Carlos I (Antártida). Trabajo Fin de Grado. Universidad de Alcalá, Facultad de Ciencias Ambientales, Madrid (inédito).

King, J.C. y Turner, J. 1997. Antarctic meteorology and climatology. Cambridge University Press, Cambridge, 409 pp.

Köppen, W. 1918. Klassifikation der Klimate nach Temperatur, Niederschlag und Jahresablauf (Classification of climates according to temperature, precipitation and seasonal cycle). Petermanns Geographische Mitteilungen., 64, 193-203, 243-248

Lewkowicz, A.G. 2008. Evaluation of miniature temperature-loggers to monitor snowpack evolution at mountain permafrost sites, northwestern Canada. Permafrost and Periglacial Processes, 19(3), 323-331.

LIE-Ropero, M.A. 2015. Proyecto de estudio de afecciones morfológicas en la base Gabriel de Castilla en isla Decepción - Antártida. Laboratorio de Ingenieros del Ejército "General Marvá", INTA - Ministerio de Defensa, Madrid. 264 pp.

Linell, K.A y, Tedrow, J.C.F. 1981. Soil and permafrost surveys in the Arctic. Oxford: Oxford University Press, 279 pp.

López-Martínez, J. y Serrano, E. 2002. Geomorphology. En: López-Martínez, J., Smellie, J., Thomson, J.W. and Thomson, M.R.A. (Eds.), Geology and geomorphology of Deception Island. BAS GEOMAP Series, Sheets 6-A and 6-B, British Antarctic Survey. Cambridge, UK. 11-30.

López-Martínez, J., Serrano, E., Rey, J., y Smellie, J.L. 2000. Geomorphological map of Deception Island. E. 1:25.000. En: López-Martínez, J., Smellie, J., Thomson, J.W. and Thomson, M.R.A. (Eds.), Geology and geomorphology 
de Pablo, M.A., et al., 2017. Análisis del estado de la capa activa en el emplazamiento... Boletín Geológico y Minero, 128 (1): $69-92$

of Deception Island. BAS GEOMAP Series, Sheets 6-A and 6-B, British Antarctic Survey. Cambridge, UK.

Maestro, A., Somoza, L., Rey, J., Martínez-Frías, J. y LópezMartínez, J. 2007. Active tectonics, fault patterns, and stress field of Deception Island: A response to oblique convergence between the Pacific and Antarctic plates. Journal of South American Earth Sciences, 23, 256-268.

Matsuoka, N. 2006. Monitoring periglacial processes: towards construction of a global network. Geomorphology, 80, 20-31.

Matsuoka, N. y Humlum, O. 2003. Monitoring periglacial processes: new methodology and technology. Permafrost and Periglacial Procceses, 14, 299-303.

Muller, S.W. 1943. Permafrost or permanently frozen ground and related engineering problems. Special Report, Strategic Engineering Study, Intelligence Branch, Office, Chief of Engineers, 62, 136 pp. Second Printing, 1945, 230 pp. (Reprinted in 1947, J.W. Edwards, Ann Arbor, Michigan, 231 pp.).

Nelson, F.E., Shiklomanov, N.I., Hinkel, K. y Christiansen, H. 2004. Introduction: The Circumpolar Active Layer Monitoring Network (CALM) workshop and CALM II program. Polar Geography, 28, 253-266.

Oht, M. 2003. Impact of meteorological factors on active layer development in Central Spitsbergen. En: Permafrost. (Eds.) Phillips, Springman and Arenson, Zurich, Switzerland, 845-850.

Oliva, M. y Ruiz-Fernández, J. 2015. Coupling patterns between para-glacial and permafrost degradation responses in Antarctica. Earth Surface Processes and Landforms, 40(9), 1227-1238.

Osterkamp, T.E. 2003. Establishing Long-term permafrost observatories for active-layer and permafrost investigations in Alaska: 1977-2002. Permafrost and Periglacial Processes, 14, 331-342.

Peel, M.C., Finlayson, B.L. y McMahon, T.A. 2007. Updated world map of the Köppen-Geiger climate classification. Hydrology and Earth System Sciences, 11, 1633-1644.

Péwé, T.L. 1983. Geologic hazards of the Fairbanks Area, Alaska. Alaska Geological and Geophysical Surveys Special Report, 15.

Ramos M., Vieira G., Guilichinski D. y de Pablo MA. 2010. Nuevas estaciones de medida del régimen térmico del permafrost en el área de Crater Lake. Isla Decepción (Antártida). Resultados preliminares. Proceedings of II Iberian Conference of the International Permafrost Association Periglacial, environments, permafrost and climate variability. En: Blanco, J.J., Ramos, M. y de Pablo, M.A. Universidad de Alcalá, Madrid, España. 93-109.

Ramos, M., de Pablo, M.A., Sebastian, E., Armiens, C. y Gómez-Elvira, J. 2012. Temperature gradient distribution in permafrost active layer, using a prototype of the ground temperature sensor (REMS-MSL) on Deception Island (Antarctica). Cold Regions Science and Technology, 72, 23-32.

Ramos, M., Ortiz, R., Diez-Gil, J.L. y Viramonte, J.G. 1989. Anomalías Térmicas y Balance del Flujo disipado en la isla Decepción, Shetland del Sur. III Simposio de Estudios Antárticos, CICYT, Madrid. 203-219.
Ramos, M., Vieira, G., Gruber, S., Blanco, J.J., Hauck, C., Hidalgo, M.A., Tomé, D., Neves, M. y Trindade, A. 2007. Permafrost and active layer monitoring in the Maritime Antarctic: Preliminary results from CALM sites on Livingston and Deception Islands. 10th International Symposium on Antarctic Earth Sciences. Abstract 1047 srp070.

Recio, C. 2015. Estudio de la erosión costera en el emplazamiento de la Base Antártica Española "Gabriel de Castilla" (isla Decepción, Antártida) y propuestas de actuaciones de restauración". Proyecto Fin de Máster. Universidad de Alcalá, Madrid. 88 pp.

Rey, J. y Somoza, L. 1992. Neotectonic of extensional processes related to the Bransfield Rift, Deception Island (Antarctica). En: Mörner, N.A., Owen, L.A. and Vita-Finzi, C. (Eds.), Neotectonics-Recent Advances. Quaternary Research Association, Cambridge, 99, 57-58.

Rey, J., Somoza, L. y Martínez-Frías, J. 1995. Tectonic, volcanic and hydrothermal event sequence on Deception Island (Antarctica). Geo-Marine Letters, 15, 1-8.

Ropero, M.A. 2010a. Módulo de vida: manual descriptivo. Plan de mantenimiento BAE Gabriel de Castilla. Órgano técnico de apoyo a instalaciones, Ministerio de Defensa, Madrid. 8 pp.

Ropero, M.A. 2010b. Módulo de dormitorios: manual descriptivo. Plan de mantenimiento BAE Gabriel de Castilla. Órgano técnico de apoyo a instalaciones, Ministerio de Defensa, Madrid. 9 pp.

Ropero, M.A. 2013. Bases antárticas: infraestructuras en condiciones extremas. Trabajo Tutelado de Investigación. Escuela Politécnica Superior del Ejército, Madrid. $116 \mathrm{pp}$.

Schoeneich, P., Dall'Amico, M., Deline, P. y Zischg A. (Eds). 2011. Hazards related to permafrost and to permafrost degradation. PermaNET project, state-of-the-art report 6.2. On-line publication ISBN 978- 2-903095-59-8. [www.permanet-alpinespace.eu]

Smellie, J.L. 2001. Litostratigraphy and volcanic evolution of Deception Island, South Shetland Islands. Antarctic Science, 13, 188-209.

Smellie, J.L. 2002. Geology. En: López-Martínez, J., Smellie, J., Thomson, J.W. and Thomson, M.R.A. (Eds.), Geology and geomorphology of Deception Island. BAS GEOMAP Series, Sheets 6-A and 6-B, British Antarctic Survey. Cambridge, UK.

Smellie, J.L. y López-Martínez, J. 2000. Geological map of Deception Island. E. 1:25.000. En: López-Martínez, J., Smellie, J., Thomson, J.W. and Thomson, M.R.A. (Eds.), Geology and geomorphology of Deception Island. BAS GEOMAP Series, Sheets 6-A and 6-B, British Antarctic Survey. Cambridge, UK.

Somoza, L., Martínez-Frías, J., Smellie, J.L., Rey, J. y Maestro, A., 2004. Evidence for hydrothermal venting and sediment volcanism discharged after recent short-lived volcanic eruptions at Deception Island, Bransfield Strait, Antarctica. Marine Geology, 203, 119-140.

Steig, E.J., Schneider, D.P., Rutherford, S.D., Mann, M.E., Comiso, J.C. and Shindell, D.T. 2009. Warming of the Antarctic ice-sheet surface since the 1957 International 
Geophysical Year. Nature, 457:7228. 459-U4. 10.1038/ nature07669.

U.S. Arctic Research Commission Permafrost Task Force. 2003. Climate Change, Permafrost, and Impacts on Civil Infrastructure. Special Report 01-03, U.S. Arctic Research Commission, Arlington, Virginia.

Van Everdingen, R.O. 1998. Multi-languaje glossary of permafrost and related ground-ice terms. International Permafrost Association (IPA), 137 pp.

Vieira, G., Bockheim, J., Guglielmin, M., Balks, M., Abramov, A.A., Boelhouwers, J., Cannone, N., Ganzert, L., Gilichinsky, D., Goryachkin, S., López-Martínez, J., Raffi, R., Ramos, M., Schaefer, C., Serrano, E., Simas,
F., Sletten, R. y Wagner, D. 2010. Thermal state of permafrost and active-layer monitoring in the Antarctic: advances during the international polar year 2007-2008. Permafrost and Periglacial Processes, 21, 182-197.

Williams, P.J. 1986. Pipelines and Permafrost: Science in a Cold Climate. Carleton University Press, Don Mills, Ontario.

Zhang, T. 2005. Influence of the seasonal snow cover on the ground thermal regime: An overview. Reviews in Geophysics, 43. RG4002. doi: 10.1029/2004RG000157

Zhao-ping, Y., Hua Ou, Y., Xu, X.-L., Zhao, L., Song, M.-H. y Zhou, C.-P. 2010. Effects of permafrost degradation on ecosystems. Acta Ecologica Sinica, 30(1), 33-39.

Recibido: noviembre 2015

Revisado: febrero 2016

Aceptado: marzo 2016

Publicado: marzo 2017 
